\title{
Entanglement entropy for descendent local operators in 2D CFTs
}

\author{
Bin Chen, ${ }^{a, b, c} \mathbf{W u}$-Zhong Guo, ${ }^{d, e}$ Song $\mathbf{H e}{ }^{e, d}$ and Jie-qiang $\mathbf{W} \mathbf{u}^{a}$ \\ ${ }^{a}$ Department of Physics and State Key Laboratory of Nuclear Physics and Technology, \\ Peking University, \\ Beijing 100871, P.R. China \\ ${ }^{b}$ Collaborative Innovation Center of Quantum Matter, \\ Beijing 100871, P.R. China \\ ${ }^{c}$ Center for High Energy Physics, Peking University, \\ Beijing 100871, P.R. China \\ ${ }^{d}$ State Key Laboratory of Theoretical Physics, \\ Institute of Theoretical Physics, Chinese Academy of Science, \\ Beijing 100190, P.R. China \\ ${ }^{e}$ Yukawa Institute for Theoretical Physics, Kyoto University, \\ Kitashirakawa Oiwakecho, Sakyo-ku, Kyoto 606-8502, Japan \\ E-mail: bchen01@pku.edu.cn, wuzhong@itp.ac.cn, hesong17@gmail.com, \\ jieqiangwu@pku.edu.cn
}

AbSTRACT: We mainly study the Rényi entropy and entanglement entropy of the states locally excited by the descendent operators in two dimensional conformal field theories (CFTs). In rational CFTs, we prove that the increase of entanglement entropy and Rényi entropy for a class of descendent operators, which are generated by $\mathcal{L}^{(-)} \overline{\mathcal{L}}^{(-)}$onto the primary operator, always coincide with the logarithmic of quantum dimension of the corresponding primary operator. That means the Rényi entropy and entanglement entropy for these descendent operators are the same as the ones of their corresponding primary operator. For 2D rational CFTs with a boundary, we confirm that the Rényi entropy always coincides with the logarithmic of quantum dimension of the primary operator during some periods of the evolution. Furthermore, we consider more general descendent operators generated by $\sum d_{\left\{n_{i}\right\}\left\{n_{j}\right\}}\left(\prod_{i} L_{-n_{i}} \prod_{j} \bar{L}_{-n_{j}}\right)$ on the primary operator. For these operators, the entanglement entropy and Rényi entropy get additional corrections, as the mixing of holomorphic and anti-holomorphic Virasoro generators enhance the entanglement. Finally, we employ perturbative CFT techniques to evaluate the Rényi entropy of the excited operators in deformed CFT. The Rényi and entanglement entropies are increased, and get contributions not only from local excited operators but also from global deformation of the theory.

Keywords: Field Theories in Lower Dimensions, Conformal and W Symmetry

ARXIV EPRINT: 1507.01157 


\section{Contents}

1 Introduction 1

2 Rényi entropy of descendent operator 3

2.1 Setup in 2D CFT 3

2.2 Convention 3

2.3 Second Rényi entanglement entropy in terms of 4-pt functions 5

2.3.1 Example I: $(2,1)$ operator in minimal model 6

2.3.2 Example II: energy momentum tensor 8

2.4 2nd Rényi entropy for some descendant operators 9

$2.5 n$-th RE for generic descendent states $\quad 13$

$\begin{array}{lll}2.6 & \text { Comments in BCFT } & 16\end{array}$

$\begin{array}{llr}3 & \text { Rényi entropy in deformed CFT } & 17\end{array}$

4 Conclusion and discussion $\quad 20$

$\begin{array}{ll}\text { A Conformal transformation for descendent operator } & 22\end{array}$

\section{Introduction}

The entanglement entropy (EE) and the entanglement Rényi entropy (RE) are very helpful quantities to study global or non-local structures in quantum field theories. For example, computing topological entanglement entropy can characterize the topological order of the system [1, 2]. In [3] a relationship between the topological entanglement entropy and boundary entropy has been pointed out. And in [4] the relationship between the boundary entropy and entanglement entropy has been explored. Both the entanglement and Rényi entropes of a subsystem $A$ are defined with respect to the reduced density matrix $\rho_{A}$, which is obtained by tracing out the degrees of freedom in the complement of $A$ from the original density matrix $\rho$. Formally, the $n$-th Rényi entanglement entropy is defined by $S_{A}^{(n)}=\log \operatorname{Tr}\left[\rho_{A}^{n}\right] /(1-n)$. If the limit $n \rightarrow 1$ is well-defined, $\lim _{n \rightarrow 1} S_{A}^{(n)}$ coincides with the von-Neumann entropy exactly, which defines the entanglement entropy. This leads to standard replica trick to in compute the entanglement entropy in the quantum field theory.

There could be topological contribution to the entanglement entropy even for gapless theories, e.g. conformal field theories (CFTs). In 2D rational CFTs, it was found [15] that for the locally primary excited states, the Rényi entropy difference is related to the quantum dimension [5-9] of the primary operator, which is a kind of topological quantity. The computations of the entanglement entropies for locally excited states have been formulated in $[10-12]$ in the field theory side. The entanglement entropy for local free scalar have 
been investigated in [12, 13] (see also [14] for the operator approach). In the large $N$ CFTs , the entanglement entropies for locally excited states have been discussed in [1618]. In a more recent paper [19], the Rényi entropy difference $\Delta S_{A}^{(n)}$ of the local excited states in a boundary conformal field theory (BCFT) has been studied. In [20] the left-right entanglement entropy for a boundary state has been discussed.

In all the study of quantum entanglement of local operator, only the primary operators have been discussed. ${ }^{1}$ It is interesting to investigate the similar effect from a local descendent operators. Among the descendants, the stress tensor is of particular interest, as it is related to the energy density in the field theory, and is dual to a graviton in the holographic dual. In this paper, we generalize the previous study $[15,19]$ on the Rényi entropy difference to the descendent operators with or without a boundary condition. First of all, we compute $\Delta S^{(2)}$ explicitly for some simple descendent operators in minimal models, and we find that in these cases the entropy difference is exactly the logarithmic of the quantum dimension of corresponding primary operator. Moreover, we study the descendant operators in a general theory. The picture is that if the local operator is of the form $\mathcal{L}^{(-)} \overline{\mathcal{L}}^{(-)} \mathcal{O}$, the entropy difference is the same as the one for the local primary operator. This could be explained by studying the divergent terms in the correlation function. However for a more generic operator like

$$
V=\sum_{\left\{n_{i}\right\},\left\{\bar{n}_{j}\right\}} d_{\left\{n_{i}\right\},\left\{\bar{n}_{j}\right\}} \prod_{i, j} L_{-n_{i}} \bar{L}_{-n_{j}} O(w, \bar{w})
$$

we find that the Rényi entropies and entanglement entropy have additional contribution. Such additional correction originates from the entanglement in the excited states. We give a systematic recipe to calculate the entanglement entropy for general descendant operators. We also investigate the Rényi entropy for the states excited by a local descendent operator in the rational CFTs with a boundary. The boundaries introduced here do not break the conformal symmetry. Such kinds of theories are called the boundary conformal field theories (BCFT). We find that the Rényi entropies excited by the descendants in BCFTs are similar to the one discussed in $[15,19]$. If the descendent states are generated by the operator (1.1), the boundary does change the time evolution of the Rényi entropy but it does not change the maximal value of the Rényi entropy. Finally, we investigate the Rényi entropies in a deformed CFT perturbatively. If the deformation can be seen as a perturbation, the Rényi entropies of locally excited states are just the summation of contributions from the local excitation and the one from global deformation.

The layout of this paper is as follows. In section 2, we would like to study the Rényi entropy of the local descendent state with or without a boundary. In section 3 , we move to study the Rényi entropy of the local excited states in 2D CFT with deformation by chemical potentials. In these deformed theories, we obtain the Rényi entropy of a subsystem with time evolution. In section 4, we devote to the conclusions and discussions. In appendix, we list some technics which are very useful in our analysis.

While proceeding with this project, we noticed a recent papers [40] appearing in arXiv, which has overlap with our discussion in the sections 2.3 and 2.4.

\footnotetext{
${ }^{1}$ There has been some discussion on the entanglement entropy for descendant excited states in [11].
} 


\section{Rényi entropy of descendent operator}

\subsection{Setup in 2D CFT}

Consider an excited state which is defined by acting a primary or a descendent operator $V_{a}$ on the vacuum $|0\rangle$ in a two dimensional CFT. We make use of the Euclidean formulation and introduce the complex coordinate $(w, \bar{w})=(x+i \tau, x-i \tau)$ on $R^{2}$ such that $\tau$ and $x$ are the Euclidean time and the space respectively. We insert the operator $V_{a}$ at $x=-l<0$ and consider its real time-evolution from time 0 to $t$ under the Hamiltonian $H$. The corresponding density matrix can be expressed as following:

$$
\begin{aligned}
\rho(t) & =\mathcal{N} \cdot e^{-i H t} e^{-\epsilon H} V_{a}(-l)|0\rangle\langle 0| V_{a}^{\dagger}(-l) e^{-\epsilon H} e^{i H t} \\
& =\mathcal{N} \cdot V_{a}\left(w_{2}, \bar{w}_{2}\right)|0\rangle\langle 0| V_{a}^{\dagger}\left(w_{1}, \bar{w}_{1}\right),
\end{aligned}
$$

where $\mathcal{N}$ is fixed by requiring $\operatorname{Tr} \rho(t)=1$, and

$$
\begin{array}{ll}
w_{1}=i(\epsilon-i t)-l, & w_{2}=-i(\epsilon+i t)-l, \\
\bar{w}_{1}=-i(\epsilon-i t)-l, & \bar{w}_{2}=i(\epsilon+i t)-l .
\end{array}
$$

The infinitesimal positive parameter $\epsilon$ is an ultraviolet regularization factor. We treat $\epsilon \pm i t$ as purely imaginary numbers until the end of the calculations, as in $[12,13,21]$.

To calculate $\Delta S_{A}^{(n)}$, we can apply the replica method in the path-integral formalism by generalizing the formulation for the ground state [4] to our excited states [12]. It leads to a $n$-sheeted Riemann surface $\Sigma_{n}$ with $2 n$ operators $V_{a}$ inserted. We choose the subsystem $A$ to be an interval $0 \leq x \leq L$ at $\tau=0$. In section 2.2, 2.3, we would like to take $L \rightarrow \infty$ as a warm up. For later parts, we will consider the situation with finite $L$.

Finally, the $\Delta S_{A}^{(n)}$ can be computed as

$$
\begin{gathered}
\Delta S_{A}^{(n)}=\frac{1}{1-n}\left[\log \left\langle V_{a}^{\dagger}\left(w_{1}, \bar{w}_{1}\right) V_{a}\left(w_{2}, \bar{w}_{2}\right) \cdots V_{a}\left(w_{2 n}, \bar{w}_{2 n}\right)\right\rangle_{\Sigma_{n}}\right. \\
\left.-n \log \left\langle V_{a}^{\dagger}\left(w_{1}, \bar{w}_{1}\right) V_{a}\left(w_{2}, \bar{w}_{2}\right)\right\rangle_{\Sigma_{1}}\right]
\end{gathered}
$$

where $\left(w_{2 k+1}, w_{2 k+2}\right)$ for $k=1,2, \ldots, n-1$ are $n-1$ replicas of $\left(w_{1}, w_{2}\right)$ in the $k$-th sheet of $\Sigma_{n}$. The term in the first line is given by a $2 n$-point correlation function on $\Sigma_{n}$, while the one in the second line is given by $n$ two-point functions on $\Sigma_{1}$. Here $\Delta_{a}$ is the (chiral and anti-chiral) conformal dimension of the operator $O_{a}$.

\subsection{Convention}

Let us study the second Rényi entanglement entropy in details to set up the convention. The calculations of $\Delta S_{A}^{(2)}$ is related to the four-point functions which we know pretty well for exactly solvable CFTs.

To compute $\Delta S_{A}^{(2)}$ we need to introduce the coordinate $z_{i}$, which is related to $w_{i}$ by the conformal map $w_{i}=z_{i}^{2}$. First the coordinates $w_{i}$ and $z_{i}$ behave like

$$
w_{1}=i \epsilon+t-l \equiv r e^{i \theta_{1}}=\left(z_{1}\right)^{2},
$$




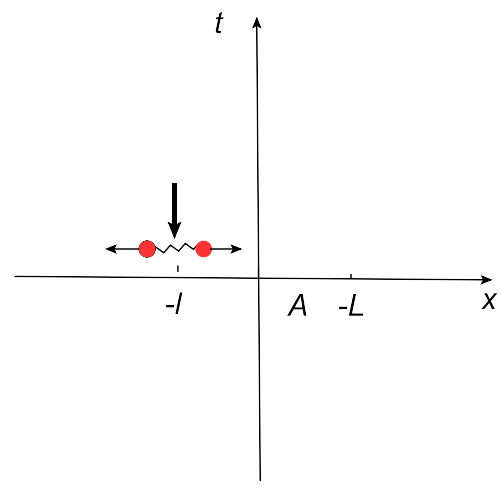

Figure 1. This figure is to show our setup in two dimensional plane $\omega=x+i t$. The system will be triggered at $x=-l$ and there are left- and right-moving quasi-particles at $t=0$.

$$
\begin{aligned}
& w_{2}=-i \epsilon+t-l \equiv s e^{i \theta_{2}}=\left(z_{2}\right)^{2}, \\
& w_{3}=(i \epsilon+t-l) e^{2 \pi i} \equiv r e^{i\left(2 \pi+\theta_{1}\right)}=\left(z_{3}\right)^{2}, \\
& w_{4}=(-i \epsilon+t-l) e^{2 \pi i} \equiv s e^{i\left(2 \pi+\theta_{2}\right)}=\left(z_{4}\right)^{2} .
\end{aligned}
$$

Thus we find

$$
\begin{aligned}
& z_{1}=-z_{3}=\sqrt{w_{1}}=\sqrt{r} e^{i \theta_{1} / 2}=i \sqrt{l-t-i \epsilon} \\
& z_{2}=-z_{4}=\sqrt{w_{2}}=\sqrt{s} e^{i \theta_{2} / 2}=i \sqrt{l-t+i \epsilon} .
\end{aligned}
$$

The most important point is that we should treat $\pm i \epsilon+t$ as a pure imaginary number in all algebraic calculations and take $t$ to be real only in the final expression of the entropy. This is a standard prescription to make analytical continuation of Euclidean theory into its Lorentzian version. Therefore we should identify

$$
\left(r \cos \theta_{1}, r \sin \theta_{1}\right)=(-l, \epsilon-i t), \quad\left(s \cos \theta_{2}, s \sin \theta_{2}\right)=(-l,-\epsilon-i t) .
$$

This leads to

$$
\begin{array}{rlrl}
r & =\sqrt{l^{2}+(\epsilon-i t)^{2}}, & s=\sqrt{l^{2}+(-\epsilon-i t)^{2}}, \\
r s & =\sqrt{\left(l^{2}+\epsilon^{2}-t^{2}\right)^{2}+4 \epsilon^{2} t^{2}}, & r^{2}+s^{2}=2\left(l^{2}+\epsilon^{2}-t^{2}\right), \\
\cos \left(\theta_{1}-\theta_{2}\right) & =2 \cos ^{2}\left(\frac{\theta_{1}-\theta_{2}}{2}\right)-1=\frac{l^{2}-\epsilon^{2}-t^{2}}{\sqrt{\left(l^{2}+\epsilon^{2}-t^{2}\right)^{2}+4 \epsilon^{2} t^{2}}} .
\end{array}
$$

To evaluate the four point-correlation functions, it is useful to focus on the ratio

$$
\begin{aligned}
& z=\frac{z_{12} z_{34}}{z_{13} z_{24}}=\frac{-(l-t)+\sqrt{(l-t)^{2}+\epsilon^{2}}}{2 \sqrt{(l-t)^{2}+\epsilon^{2}}}, \\
& \bar{z}=\frac{\bar{z}_{12} \bar{z}_{34}}{\bar{z}_{13} \bar{z}_{24}}=\frac{-(l+t)+\sqrt{(l+t)^{2}+\epsilon^{2}}}{2 \sqrt{(l+t)^{2}+\epsilon^{2}}},
\end{aligned}
$$

where $z_{i j}=z_{i}-z_{j}$. Note that there is a useful relation

$$
1-z=\frac{z_{14} z_{23}}{z_{13} z_{24}}
$$


We are interested in the two limits (i) $l \gg t \gg \epsilon$ (early time) and (ii) $t \gg l \gg \epsilon$ (late time). From (2.9) we find that they correspond to

$$
\begin{aligned}
& \text { (i) } z \simeq \bar{z} \simeq \frac{\epsilon^{2}}{4 l^{2}}(\rightarrow 0), \\
& \text { (ii) } z \simeq 1-\frac{\epsilon^{2}}{4 t^{2}}(\rightarrow 1), \quad \bar{z} \simeq \frac{\epsilon^{2}}{4 t^{2}}(\rightarrow 0) .
\end{aligned}
$$

Note that the second limit is non-trivial in that it does not respect the complex conjugate relation because of our analytical continuation of $t$.

\subsection{Second Rényi entanglement entropy in terms of 4-pt functions}

Let us first consider the Rényi entropy of the simplest descendent operator $L_{-1} O$, where $O$ is a primary field. The four-point function on $n$-sheeted Riemann surface $\Sigma_{n}$ can be mapped to the one on $R^{2}$ by the conformal map $w=z^{n}$. For the second Rényi entropy, we find

$$
\begin{aligned}
&\left\langle L_{-1} O\left(w_{1}, \bar{w}_{1}\right) L_{-1} O\left(w_{2}, \bar{w}_{2}\right) L_{-1} O\left(w_{3}, \bar{w}_{3}\right) L_{-1} O\left(w_{4}, \bar{w}_{4}\right)\right\rangle_{\Sigma_{2}} \\
&=\prod_{i=1}^{4}\left|\frac{d w_{i}}{d z_{i}}\right|^{-2 \Delta}\left(\left\langle\left(-\Delta \frac{\partial z_{1}}{\partial w_{1}} \frac{\partial^{2} w_{1}}{\partial z_{1}^{2}}+\frac{\partial z_{1}}{\partial w_{1}} \partial_{z_{1}}\right) O\left(z_{1}, \bar{z}_{1}\right)\left(-\Delta \frac{\partial z_{2}}{\partial w_{2}} \frac{\partial^{2} w_{2}}{\partial z_{2}^{2}}+\frac{\partial z_{2}}{\partial w_{2}} \partial_{z_{2}}\right) O\left(z_{2}, \bar{z}_{2}\right)\right.\right. \\
&\left.\left.\times\left(-\Delta \frac{\partial z_{3}}{\partial w_{3}} \frac{\partial^{2} w_{3}}{\partial z_{3}^{2}}+\frac{\partial z_{3}}{\partial w_{3}} \partial_{z_{3}}\right) O\left(z_{3}, \bar{z}_{3}\right)\left(-\Delta \frac{\partial z_{4}}{\partial w_{4}} \frac{\partial^{2} w_{4}}{\partial z_{4}^{2}}+\frac{\partial z_{4}}{\partial w_{4}} \partial_{z_{4}}\right) O\left(z_{4}, \bar{z}_{4}\right)\right\rangle_{\Sigma_{1}}\right) \\
&=2^{-8 \Delta}\left|z_{1} z_{2} z_{3} z_{4}\right|^{-2 \Delta} \cdot N_{1} N_{2} N_{3} N_{4}\left(\Delta^{4}\left\langle O\left(z_{1}, \bar{z}_{1}\right) O\left(z_{2}, \bar{z}_{2}\right) O\left(z_{3}, \bar{z}_{3}\right) O\left(z_{4}, \bar{z}_{4}\right)\right\rangle_{\Sigma_{1}}\right. \\
&-\Delta^{3} \sum_{i=1}^{4} \frac{M_{i}}{N_{i}} \partial_{z_{i}}\left\langle O\left(z_{1}, \bar{z}_{1}\right) O\left(z_{2}, \bar{z}_{2}\right) O\left(z_{3}, \bar{z}_{3}\right) O\left(z_{4}, \bar{z}_{4}\right)\right\rangle_{\Sigma_{1}} \\
&+\Delta^{2} \sum_{i \neq j}^{4} \frac{M_{i} M_{j}}{N_{i} N_{j}} \partial_{z_{i}} \partial_{z_{j}}\left\langle O\left(z_{1}, \bar{z}_{1}\right) O\left(z_{2}, \bar{z}_{2}\right) O\left(z_{3}, \bar{z}_{3}\right) O\left(z_{4}, \bar{z}_{4}\right)\right\rangle_{\Sigma_{1}} \\
&-\Delta \sum_{i \neq j \neq k}^{4} \frac{M_{i} M_{j} M_{k}}{N_{i} N_{j} N_{k}} \partial_{z_{i}} \partial_{z_{j}} \partial_{z_{k}}\left\langle O\left(z_{1}, \bar{z}_{1}\right) O\left(z_{2}, \bar{z}_{2}\right) O\left(z_{3}, \bar{z}_{3}\right) O\left(z_{4}, \bar{z}_{4}\right)\right\rangle_{\Sigma_{1}}+ \\
&\left.+\frac{M_{1} M_{2} M_{3} M_{4}}{N_{1} N_{2} N_{3} N_{4}} \partial_{z_{1}} \partial_{z_{2}} \partial_{z_{3}} \partial_{z_{4}}\left\langle O\left(z_{1}, \bar{z}_{1}\right) O\left(z_{2}, \bar{z}_{2}\right) O\left(z_{3}, \bar{z}_{3}\right) O\left(z_{4}, \bar{z}_{4}\right)\right\rangle_{\Sigma_{1}}\right)
\end{aligned}
$$

where $\Delta$ is the chiral conformal dimension of the primary operator $O$, and

$$
N_{i}=\frac{\partial z_{i}}{\partial w_{i}} \frac{\partial^{2} w_{i}}{\partial z_{i}^{2}}, \quad M_{i}=\frac{\partial z_{i}}{\partial w_{i}} .
$$

Since $N_{i}$ and $M_{i}$ do not contribute to the divergent terms in both the late time limit and the early time limit, we can take them as a factor to simplify our analysis. In (2.12), the last term in the summation diverge fastest in the small $\epsilon$ limit, so we only keep this term in the later calculation. Note also that $r s$ behaves in the two limits as

(i) Early time $: r s \simeq l^{2}$,

(ii) Late time $: r s \simeq t^{2}$. 
Due to the conformal symmetry, the four-point function on $R^{2}$ can be expressed as

$$
\left\langle O\left(z_{1}, \bar{z}_{1}\right) O\left(z_{2}, \bar{z}_{2}\right) O\left(z_{3}, \bar{z}_{3}\right) O\left(z_{4}, \bar{z}_{4}\right)\right\rangle_{\Sigma_{1}}=\left|z_{13} z_{24}\right|^{-4 \Delta} \cdot G(z, \bar{z}),
$$

where $(z, \bar{z})$ are given by $(2.9)$.

The two-point function is

$$
\left\langle L_{-1} O\left(w_{1}, \bar{w}_{1}\right) L_{-1} O\left(w_{2}, \bar{w}_{2}\right)\right\rangle_{\Sigma_{1}}=\partial_{w_{1}} \partial_{w_{2}} \frac{\mathcal{N}}{\left|w_{12}\right|^{4 \Delta}}=\frac{2 \Delta(2 \Delta+1) \mathcal{N}}{(2 \epsilon)^{4 \Delta+2}},
$$

where $\mathcal{N}$ is the normalization of the primary operator $O$. Note that the four-point function is proportional to $\mathcal{N}^{2}$ and the $\Delta S_{A}^{(2)}$ is of course independent of $\mathcal{N}$.

In the late time limit (ii), we finally find that the ratio $Z_{2} /\left(Z_{1}\right)^{2}$ in (2.4) is expressed in terms of the four-point function on $R^{2}$ :

$$
\begin{aligned}
& \operatorname{Tr} \rho_{A}^{2}=\frac{\left\langle L_{-1} O\left(w_{1}, \bar{w}_{1}\right) L_{-1} O\left(w_{2}, \bar{w}_{2}\right) L_{-1} O\left(w_{3}, \bar{w}_{3}\right) L_{-1} O\left(w_{4}, \bar{w}_{4}\right)\right\rangle_{\Sigma_{2}}}{\left(\left\langle L_{-1} O\left(w_{1}, \bar{w}_{1}\right) L_{-1} O\left(w_{2}, \bar{w}_{2}\right)\right\rangle_{\Sigma_{1}}\right)^{2}} \\
& \simeq \frac{1}{(2 \Delta)^{2}(2 \Delta+1)^{2} \mathcal{N}^{2}} \cdot \frac{16 \epsilon^{8 \Delta+4}}{t^{4 \Delta}}\left(\prod_{i=1}^{4} N_{i}\right) \partial_{z_{1}} \partial_{z_{2}} \partial_{z_{3}} \partial_{z_{4}}\left\langle O\left(z_{1}, \bar{z}_{1}\right) O\left(z_{2}, \bar{z}_{2}\right) O\left(z_{3}, \bar{z}_{3}\right) O\left(z_{4}, \bar{z}_{4}\right)\right\rangle_{\Sigma_{1}} \\
& \simeq \frac{1}{\left(2 \Delta^{2}\right)(2 \Delta+1)^{2} \mathcal{N}^{2}} \cdot \frac{16 \epsilon^{8 \Delta+4}}{\left(4 t^{2}\right)^{4 \Delta}} \cdot\left(\prod_{i=1}^{4} N_{i}\right) \partial_{z_{1}} \partial_{z_{2}} \partial_{z_{3}} \partial_{z_{4}} G(z, \bar{z})
\end{aligned}
$$

\subsubsection{Example I: $(2,1)$ operator in minimal model}

Now we would like to study an explicit examples: the $(2,1)$ operator in the minimal models. Let us consider a $\left(p, p^{\prime}\right)$ minimal model with $p>p^{\prime}$, where the primary fields $\phi_{\left(r_{1}, r_{2}\right)}$ are specified by a pair of integers $\left(r_{1}, r_{2}\right)$ taking the values

$$
r_{1}=1,2, \ldots, p^{\prime}-1, \quad r_{2}=1,2, \ldots, p-1 .
$$

The central charge of $\left(p, p^{\prime}\right)$ minimal model is

$$
c=1-6 \frac{\left(p-p^{\prime}\right)^{2}}{p p^{\prime}},
$$

and the conformal dimension of the $\left(r_{1}, r_{2}\right)$ primary operator is given by

$$
\Delta_{r_{1}, r_{2}}=\frac{\left(p r_{1}-p^{\prime} r_{2}\right)^{2}-\left(p-p^{\prime}\right)^{2}}{4 p p^{\prime}} .
$$

Let us focus on the $(2,1)$ operator $\phi_{(2,1)}$ in a $\left(p, p^{\prime}\right)$ minimal model as it has a relatively simple four-point function. It has conformal dimension

$$
\Delta_{(2,1)}=\frac{3 p}{4 p^{\prime}}-\frac{1}{2} .
$$

The function $G(z, \bar{z})$ for the four-point function $\left\langle\phi_{2,1}\left(z_{1}, \bar{z}_{1}\right) \ldots \phi_{2,1}\left(z_{4}, \bar{z}_{4}\right)\right\rangle_{\Sigma_{1}}$ via the relation $(2.15)$ is known to be $[22,23]$

$$
G(z, \bar{z})=|z|^{\frac{p}{p^{\prime}}}|1-z|^{\frac{p}{p^{\prime}}} \cdot\left[\frac{\sin \left(\frac{\pi p}{p^{\prime}}\right) \sin \left(\frac{3 \pi p}{p^{\prime}}\right)}{\sin \left(\frac{2 \pi p}{p^{\prime}}\right)}\left|I_{1}(z)\right|^{2}+\frac{\sin \left(\frac{\pi p}{p^{\prime}}\right) \sin \left(\frac{\pi p}{p^{\prime}}\right)}{\sin \left(\frac{2 \pi p}{p^{\prime}}\right)}\left|I_{2}(z)\right|^{2}\right],
$$


where we have defined $[24-27]$

$$
\begin{aligned}
& I_{1}(z)=\frac{\Gamma\left(\frac{3 p}{p^{\prime}}-1\right) \Gamma\left(1-\frac{p}{p^{\prime}}\right)}{\Gamma\left(\frac{2 p}{p^{\prime}}\right)} \cdot F\left[\frac{p}{p^{\prime}},-1+\frac{3 p}{p^{\prime}}, \frac{2 p}{p^{\prime}} ; z\right], \\
& I_{2}(z)=z^{1-\frac{2 p}{p^{\prime}}} \cdot \frac{\Gamma\left(1-\frac{p}{p^{\prime}}\right) \Gamma\left(1-\frac{p}{p^{\prime}}\right)}{\Gamma\left(2-\frac{2 p}{p^{\prime}}\right)} \cdot F\left[\frac{p}{p^{\prime}}, 1-\frac{p}{p^{\prime}}, 2-\frac{2 p}{p^{\prime}} ; z\right] .
\end{aligned}
$$

with $F[a, b, c ; z]$ being the hypergeometric function. By taking the limit $z_{12}=z_{34} \rightarrow 0$, we find that

$$
\left\langle\phi_{2,1}\left(z_{1}, \bar{z}_{1}\right) \ldots \phi_{2,1}\left(z_{4}, \bar{z}_{4}\right)\right\rangle_{\Sigma_{1}} \rightarrow\left|z_{12}\right|^{-8 \Delta} \cdot \frac{\sin \left(\frac{\pi p}{p^{\prime}}\right)^{2}}{\sin \left(\frac{2 \pi p}{p^{\prime}}\right)} \cdot \frac{\Gamma\left(1-\frac{p}{p^{\prime}}\right)^{4}}{\Gamma\left(2-\frac{2 p}{p^{\prime}}\right)^{2}} .
$$

Thus we can identify the normalization factor $\mathcal{N}$ of the two-point function as follows

$$
\mathcal{N}^{2}=\frac{\sin \left(\frac{\pi p}{p^{\prime}}\right)^{2}}{\sin \left(\frac{2 \pi p}{p^{\prime}}\right)} \cdot \frac{\Gamma\left(1-\frac{p}{p^{\prime}}\right)^{4}}{\Gamma\left(2-\frac{2 p}{p^{\prime}}\right)^{2}}
$$

Now we turn to the most interesting limit: late time limit $t \gg l \gg \epsilon$, where we have $z=1-\frac{\epsilon^{2}}{4 t^{2}}$ and $\bar{z}=\frac{\epsilon^{2}}{4 t^{2}}$. Under this limit we can put (2.22) into the final formula (2.17). Notice that the $I_{2}$ term is more divergent in the small $\epsilon$ limit, and using the identities on the hypergeometric functions, we have

$$
\begin{aligned}
& I_{2}(z) \simeq(1-z)^{1-\frac{2 p}{p^{\prime}}} \frac{\Gamma\left(1-\frac{p}{p^{\prime}}\right) \Gamma\left(-1+\frac{2 p}{p^{\prime}}\right)}{\Gamma\left(\frac{p}{p^{\prime}}\right)}=\frac{\Gamma\left(1-\frac{p}{p^{\prime}}\right) \Gamma\left(-1+\frac{2 p}{p^{\prime}}\right)}{\Gamma\left(\frac{p}{p^{\prime}}\right)} \cdot\left(\frac{\epsilon^{2}}{4 t^{2}}\right)^{1-2 p / p^{\prime}}, \\
& I_{2}(\bar{z}) \simeq \bar{z}^{1-\frac{2 p}{p^{\prime}}} \frac{\Gamma\left(1-\frac{p}{p^{\prime}}\right)^{2}}{\Gamma\left(2-\frac{p}{p^{\prime}}\right)}=\frac{\Gamma\left(1-\frac{p}{p^{\prime}}\right)^{2}}{\Gamma\left(2-\frac{p}{p^{\prime}}\right)} \cdot\left(\frac{\epsilon^{2}}{4 t^{2}}\right)^{1-2 p / p^{\prime}} \cdot
\end{aligned}
$$

Thus we find

$$
G(z, \bar{z}) \simeq \frac{\sin \left(\frac{\pi p}{p^{\prime}}\right)^{2}}{\sin \left(\frac{2 \pi p}{p^{\prime}}\right)} \cdot \frac{\Gamma\left(1-\frac{p}{p^{\prime}}\right)^{3} \Gamma\left(-1+\frac{2 p}{p^{\prime}}\right)}{\Gamma\left(2-\frac{2 p}{p^{\prime}}\right) \Gamma\left(\frac{p}{p^{\prime}}\right)} \cdot\left(\frac{\epsilon^{2}}{4 t^{2}}\right)^{2-3 p / p^{\prime}} .
$$

In this way, we finally obtain

$$
\operatorname{Tr} \rho_{A}^{2}=\frac{\Gamma\left(2-\frac{2 p}{p^{\prime}}\right)^{3} \Gamma\left(-1+\frac{2 p}{p^{\prime}}\right)}{\Gamma\left(1-\frac{p}{p^{\prime}}\right) \Gamma\left(\frac{p}{p^{\prime}}\right)}=-\frac{1}{2 \cos \left(\frac{\pi p}{p^{\prime}}\right)},
$$

and the Renyi entanglement entropy difference

$$
\Delta S_{A}^{(2)}=\log \left[-2 \cos \left(\frac{\pi p}{p^{\prime}}\right)\right]
$$


For a general minimal model, the entropy difference is not vanishing for the descendent operator $L_{-1} O(2,1)$ operator. But for the Ising model $\left(p, p^{\prime}\right)=(4,3)$ [24-27], it is vanishing.

In a minimal model, the modular transformation maps the primary operator $\left(s_{1}, s_{2}\right)$ into $\left(r_{1}, r_{2}\right)$. Its S-matrix is defined to be $S_{\left(r_{1}, r_{2}\right),\left(s_{1}, s_{2}\right)}$, given explicitly by

$$
S_{\left(r_{1}, r_{2}\right),\left(s_{1}, s_{2}\right)}=2 \sigma \frac{2}{p p^{\prime}}(-1)^{1+r_{2} s_{1}+r_{1} s_{2}} \sin \left(\frac{\pi p}{p^{\prime}} r_{1} s_{1}\right) \sin \left(\frac{\pi p^{\prime}}{p} r_{2} s_{2}\right) .
$$

The quantum dimension $d_{\left(r_{1}, r_{2}\right)}$ for the primary field $\left(r_{1}, r_{2}\right)$ is defined by

$$
d_{\left(r_{1}, r_{2}\right)}=\frac{S_{(1,1),\left(r_{1}, r_{2}\right)}}{S_{(1,1),(1,1)}}=-2 \cos \left(\frac{\pi p}{p^{\prime}}\right) .
$$

Thus we can conclude that for the $L_{-1} O(2,1)$ operator

$$
\Delta S_{A}^{(2)}=\log d_{\left(r_{1}, r_{2}\right)},
$$

the same as the one for the primary operator $O(2,1)$.

\subsubsection{Example II: energy momentum tensor}

Another simple case is the excitation of energy momentum tenor, which is the descendent state of the identity operator. Different from the case discussed above, it is $L_{-2}$ rather than $L_{-1}$. We only consider $\Delta S_{2}$ here. The two-, three- and four-point correlation functions on the $R^{2}$ depend only on the central charge $c$ [28], which are respectively

$$
\begin{aligned}
\left\langle T\left(z_{1}\right) T\left(z_{2}\right)\right\rangle & =\frac{c}{2 z_{12}^{2}}, \\
\left\langle T\left(z_{1}\right) T\left(z_{2}\right) T\left(z_{3}\right) T\left(z_{4}\right)\right\rangle & =\frac{\mathcal{F}(z)}{z_{12}^{4} z_{34}^{4}},
\end{aligned}
$$

with

$$
\mathcal{F}(z)=\frac{c^{4}}{4}\left(1+z^{4}+\frac{z^{4}}{(1-z)^{4}}\right)+2 c \frac{z^{2}\left(1-z+z^{2}\right)}{(1-z)^{2}},
$$

$z=z_{12} z_{34} /\left(z_{13} z_{24}\right)$ is the ratio. The transformation of the energy momentum tensor under the map $z(\omega)$ is given by

$$
T(\omega)=\left(\frac{d z}{d \omega}\right)^{2} T(z)+\frac{c}{12}\{z, \omega\}
$$

with the Schwarzian derivative

$$
\{z, \omega\}=\left(z^{\prime \prime \prime} z^{\prime}-\frac{3}{2}\left(z^{\prime \prime}\right)^{2}\right) /\left(z^{\prime}\right)^{2} .
$$

In the coordinate (2.5) the leading order of the two-point function is

$$
\left\langle T\left(\omega_{1}\right) T\left(\omega_{2}\right)\right\rangle=\frac{1}{(2 \epsilon)^{4}} .
$$


After the transformation the four-point correlation function in the $\omega$ coordinate is

$$
\left\langle T\left(\omega_{1}\right) T\left(\omega_{2}\right) T\left(\omega_{3}\right) T\left(\omega_{4}\right)\right\rangle=\prod_{i}\left(\frac{d z_{i}}{d \omega_{i}}\right)^{2}\left\langle T\left(z_{1}\right) T\left(z_{2}\right) T\left(z_{3}\right) T\left(z_{4}\right)\right\rangle+\ldots,
$$

where '...' denotes the less divergent terms, such as the product of three-point function and the Schwarzian derivative. During the time $0<t<l$ or $t>L+l$, we can see $z_{12} \sim z_{34} \sim O(\epsilon)$, also $z \sim O\left(\epsilon^{2}\right)$. One could see from (2.34) the leading contribution of the four-point function is

$$
\left\langle T\left(z_{1}\right) T\left(z_{2}\right) T\left(z_{3}\right) T\left(z_{4}\right)\right\rangle \simeq \frac{c^{2}}{4 z_{12}^{4} z_{34}^{4}}
$$

with

$$
z_{12}=-\frac{i L \epsilon}{(t-L)(t-L-L)} z_{1}, \quad z_{34}=-\frac{i L \epsilon}{(t-L(t-L-l))} .
$$

During the time $l<t<L+l, z_{1}=-z_{2}, z_{3}=-z_{4}, z_{23} \sim z_{14} \sim O(\epsilon)$, and $z \sim 1-O\left(\epsilon^{2}\right)$, with

$$
z \simeq 1-\frac{L^{2} \epsilon^{2}}{4(t-l)^{2}(t-L-l)^{2}} .
$$

Now the leading order contribution of the four-point correlation function in the limit $\epsilon \rightarrow 0$ is

$$
\left\langle T\left(z_{1}\right) T\left(z_{2}\right) T\left(z_{3}\right) T\left(z_{4}\right)\right\rangle \simeq \frac{c^{2}}{4(1-z)^{4}} \frac{1}{\left(4 z_{1} z_{3}\right)^{4}} .
$$

One could check (2.40) is the same as (2.43). Therefore, by using (2.4), we find $\Delta S^{(2)}=0$ during the evolution. This is just as we expected, since $T(z)$ is the descendent operator of identity $I$, the quantum dimension of which is 1 .

\subsection{2nd Rényi entropy for some descendant operators}

In previous section, we computed the Rényi entropy of some specific descendent operators and found that these descendant operators had the same contribution as the primary operators. In this subsection, we use the conformal block and operator product expansion (OPE) to show this is true for a large class of descendent operators. It is also a warm up for the study of more general descendant operators in the next subsection. In this and next subsection, we will consider the case that the interval is finite, lying at $[0, L]$, and the operators is inserted at $-l$. The two-point and $2 n$-point functions on single sheet and $n$-sheeted Riemann surfaces are respectively

$$
\begin{aligned}
& Z_{1}=\left\langle V(w, \bar{w}) V\left(w^{\prime}, \bar{w}^{\prime}\right)\right\rangle \\
& Z_{n}=\left.\left\langle V\left(w_{1}, \bar{w}_{1}\right) V\left(w_{1}^{\prime}, \bar{w}_{1}^{\prime}\right) V\left(w_{2}, \bar{w}_{2}\right) V\left(w_{2}^{\prime}, \bar{w}_{2}^{\prime}\right) \ldots V\left(w_{n}, \bar{w}_{n}\right) V\left(w_{n}^{\prime}, \bar{w}_{n}^{\prime}\right)\right\rangle\right|_{n-\text { sheets }},
\end{aligned}
$$

where the correlation function $Z_{n}$ is defined on the $n$-sheeted surface, with $V\left(w_{i}, \bar{w}_{i}\right)$ on the $i$-th surface. We set

$$
w_{i}=-l+(t-i \epsilon), \quad \bar{w}_{i}=-l+(t+i \epsilon),
$$




$$
w_{i}^{\prime}=-l+(t+i \epsilon), \quad \bar{w}_{i}^{\prime}=-l-(t+i \epsilon) .
$$

$V(w, \bar{w})$ can be any descendant operators. ${ }^{2}$ We only need to set $n=2$ for $S^{(2)}$ calculation. To evaluate the multi-point correlation function on $n$-sheeted surface, we need to take a conformal transformation

$$
z=\left(\frac{w}{w-L}\right)^{\frac{1}{n}} .
$$

First we consider the descendent operator $V(w, \bar{w})=\partial O_{a}(w, \bar{w})$, where $O_{a}(w, \bar{w})$ is a primary operator, the two-point function equals to

$$
Z_{1}=\left\langle\partial O_{a}(w, \bar{w}) \partial O_{a}\left(w^{\prime}, \bar{w}^{\prime}\right)\right\rangle=-2 h(2 h+1) \frac{1}{\left(w-w^{\prime}\right)^{2 h+2}} \frac{1}{\left(\bar{w}-\bar{w}^{\prime}\right)^{2 h}}=\frac{2 h(2 h+1)}{(2 \epsilon)^{4 h+2}},
$$

and the four-point function is

$$
Z_{2}=\partial_{w_{1}} \partial_{w_{1}^{\prime}} \partial_{w_{2}} \partial_{w_{2}^{\prime}}\left\langle O_{a}\left(w_{1}, \bar{w}_{1}\right) O_{a}\left(w_{1}^{\prime}, \bar{w}_{1}^{\prime}\right) O_{a}\left(w_{2}, \bar{w}_{2}\right) O_{a}\left(w_{2}^{\prime}, \bar{w}_{2}^{\prime}\right)\right\rangle
$$

Under the conformal transformation (2.48), for $t<l$

$$
\begin{aligned}
z_{1} & =\frac{(l-t+i \epsilon)^{\frac{1}{2}}}{(l+L-t+i \epsilon)^{\frac{1}{2}}}, & \bar{z}_{1} & =\frac{(l+t-i \epsilon)^{\frac{1}{2}}}{(L+l+t-i \epsilon)^{\frac{1}{2}}}, \\
z_{1}^{\prime} & =\frac{(l-t-i \epsilon)^{\frac{1}{2}}}{(l+L-t-i \epsilon)^{\frac{1}{2}}}, & \bar{z}_{1}^{\prime} & =\frac{(l+t+i \epsilon)^{\frac{1}{2}}}{(l+L+t+i \epsilon)^{\frac{1}{2}}} \\
z_{2} & =-\frac{(l-t+i \epsilon)^{\frac{1}{2}}}{(l+L-t+i \epsilon)^{\frac{1}{2}}} & \bar{z}_{2} & =-\frac{(l+t-i \epsilon)^{\frac{1}{2}}}{(L+l+t-i \epsilon)^{\frac{1}{2}}} \\
z_{2}^{\prime} & =-\frac{(l-t-i \epsilon)^{\frac{1}{2}}}{(l+L-t-i \epsilon)^{\frac{1}{2}}} & \bar{z}_{2}^{\prime} & =-\frac{(l+t+i \epsilon)^{\frac{1}{2}}}{(l+L+t+i \epsilon)^{\frac{1}{2}}},
\end{aligned}
$$

where $\left(z_{1}, z_{1}^{\prime}\right)\left(z_{2}, z_{2}^{\prime}\right)\left(\bar{z}_{1}, \bar{z}_{1}^{\prime}\right)\left(\bar{z}_{2}, \bar{z}_{2}^{\prime}\right)$ are close to each other. While for $l<t<l+L$,

$$
\begin{aligned}
& z_{1}=-z_{2}=e^{\frac{\pi i}{2}} \frac{(t-l-i \epsilon)^{\frac{1}{2}}}{(l+L-t+i \epsilon)^{\frac{1}{2}}} \\
& z_{1}^{\prime}=-z_{2}^{\prime}=e^{-\frac{\pi i}{2}} \frac{(t-l+i \epsilon)^{\frac{1}{2}}}{(l+L-t-i \epsilon)^{\frac{1}{2}}}
\end{aligned}
$$

then $\left(z_{1}, z_{2}^{\prime}\right)\left(z_{2}, z_{1}^{\prime}\right)\left(\bar{z}_{1}, \bar{z}_{1}^{\prime}\right)\left(\bar{z}_{2}, \bar{z}_{2}^{\prime}\right)$ are close to each other.

From the result in [15], for $t<l$,

$$
\begin{aligned}
& \left\langle O_{a}\left(z_{1}, \bar{z}_{1}\right) O_{a}\left(z_{1}^{\prime}, \bar{z}_{1}^{\prime}\right) O_{a}\left(z_{2}, \bar{z}_{2}\right) O_{a}\left(z_{2}^{\prime}, \bar{z}_{2}^{\prime}\right)\right\rangle \\
& =\frac{1}{\left(z_{1}-z_{1}^{\prime}\right)^{2 h}\left(\bar{z}_{1}-\bar{z}_{1}^{\prime}\right)^{2 h}} \frac{1}{\left(z_{2}-z_{2}^{\prime}\right)^{2 h}\left(\bar{z}_{2}-\bar{z}_{2}^{\prime}\right)^{2 h}}+\text { less divergent term }
\end{aligned}
$$

\footnotetext{
${ }^{2}$ For convenience the convention in this section and the next section is a little different from the one in other sections. The correspondence is $w_{2 j} \rightarrow w_{j}, w_{2 j-1} \rightarrow w_{j}^{\prime}$.
} 
and for $l<t<l+L$,

$$
\begin{aligned}
& \left\langle O_{a}\left(z_{1}, \bar{z}_{1}\right) O_{a}\left(z_{1}^{\prime}, \bar{z}_{1}^{\prime}\right) O_{a}\left(z_{2}, \bar{z}_{2}\right) O_{a}\left(z_{2}^{\prime}, \bar{z}_{2}^{\prime}\right)\right\rangle \\
& =\frac{1}{d_{a}} \frac{1}{\left(z_{1}-z_{2}^{\prime}\right)^{2 h}\left(\bar{z}_{1}-\bar{z}_{1}^{\prime}\right)^{2 h}} \frac{1}{\left(z_{2}-z_{1}^{\prime}\right)^{2 h}\left(\bar{z}_{2}-\bar{z}_{2}^{\prime}\right)^{2 h}}+\text { less divergent term. }
\end{aligned}
$$

Taking a conformal transformation (2.48), for $t<l$

$$
\begin{aligned}
& \left.\left\langle O_{a}\left(w_{1}, \bar{w}_{1}\right) O_{a}\left(w_{1}^{\prime}, \bar{w}_{1}^{\prime}\right) O_{a}\left(w_{2}, \bar{w}_{2}\right) O_{a}\left(w_{2}^{\prime}, \bar{w}_{2}^{\prime}\right)\right\rangle\right|_{2-\text { sheets }} \\
& =\frac{1}{\left(w_{1}-w_{1}^{\prime}\right)^{2 h}\left(\bar{w}_{1}-\bar{w}_{1}^{\prime}\right)^{2 h}} \frac{1}{\left(w_{2}-w_{2}^{\prime}\right)^{2 h}\left(\bar{w}_{2}-\bar{w}_{2}^{\prime}\right)^{2 h}}+\text { less divergent term, }
\end{aligned}
$$

and for $l<t<l+L$

$$
\begin{aligned}
& \left.\left\langle O_{a}\left(w_{1}, \bar{w}_{1}\right) O_{a}\left(w_{1}^{\prime}, \bar{w}_{1}^{\prime}\right) O_{a}\left(w_{2}, \bar{w}_{2}\right) O_{a}\left(w_{2}^{\prime}, \bar{w}_{2}^{\prime}\right)\right\rangle\right|_{2-\text { sheets }} \\
& =\frac{1}{d_{a}} \frac{1}{\left(w_{1}-w_{2}^{\prime}\right)^{2 h}\left(\bar{w}_{1}-\bar{w}_{1}^{\prime}\right)^{2 h}} \frac{1}{\left(w_{2}-w_{1}^{\prime}\right)^{2 h}\left(\bar{w}_{2}-\bar{w}_{2}^{\prime}\right)^{2 h}}+\text { less divergent term, }
\end{aligned}
$$

where we still only keep the leading divergent term for small $\epsilon$. Consequently for $l<t<$ $l+L$

$$
\begin{aligned}
& \partial_{w_{1}} \partial_{w_{1}^{\prime}} \partial_{w_{2}} \partial_{w_{2}^{\prime}}\left\langle O_{a}\left(w_{1}, \bar{w}_{1}\right) O_{a}\left(w_{1}^{\prime}, \bar{w}_{1}^{\prime}\right) O_{a}\left(w_{2}, \bar{w}_{2}\right) O_{a}\left(w_{2}^{\prime}, \bar{w}_{2}^{\prime}\right)\right\rangle \\
& =\frac{(2 h)^{2}(2 h+1)^{2}}{d_{a}} \frac{1}{\left(w_{1}-w_{2}^{\prime}\right)^{2 h+2}\left(\bar{w}_{1}-\bar{w}_{1}^{\prime}\right)^{2 h}} \frac{1}{\left(w_{2}-w_{1}^{\prime}\right)^{2 h+2}\left(\bar{w}_{2}-\bar{w}_{2}^{\prime}\right)^{2 h}}+\text { lower divergent term } \\
& =\frac{(2 h)^{2}(2 h+1)^{2}}{d_{a}} \frac{1}{(2 \epsilon)^{8 h+4}}+\text { lower divergent term, }
\end{aligned}
$$

which leads to $\Delta S_{2}=\log d_{a}$ for $l<t<l+L$.

Next we consider the descendent operators with the form

$$
V(w, \bar{w})=L^{(-)} O(w, \bar{w}),
$$

where $L^{(-)}$is a combination of holomorphic generators such that $V(w, \bar{w})$ is a quasi-primary operator. ${ }^{3}$ We also assume the operator $L^{(-)}$has a fixed conformal dimension

$$
\left[L_{0}, L^{(-)}\right]=m L^{(-)} .
$$

Because the final result only depend on the most singular term in the two-point function and the $2 n$-point function, there is nothing change by adding in the operators with smaller conformal dimensions.

The conformal transformation for the descendant operators are different from the one for the primary operator. Under a conformal transformation

$$
\begin{aligned}
\left.L_{-m}\right|_{w_{1}} & =\frac{1}{2 \pi i} \oint \frac{d w}{\left(w-w_{1}\right)^{m-1}} T(w) \\
& =\frac{1}{2 \pi i} \oint \frac{d w}{\left(w-w_{1}\right)^{m-1}}\left(T(z)\left(\frac{\partial z}{\partial w}\right)^{2}+\frac{c}{12}\{z, w\}\right)
\end{aligned}
$$

\footnotetext{
${ }^{3}$ This condition can be relaxed from the result in the next subsection, but for simplicity we still assume this condition in this subsection.
} 


$$
\begin{aligned}
& =\frac{1}{2 \pi i} \oint \frac{d w}{\left(w-w_{1}\right)^{m-1}}\left(\sum_{r} \frac{\left.L_{-r}\right|_{z_{1}}}{\left(z-z_{1}\right)^{-r+2}}\left(\frac{\partial z}{\partial w}\right)^{2}+\frac{c}{12}\{z, w\}\right) \\
& =\left.L_{-m}\right|_{z_{1}}\left(\frac{\partial z_{1}}{\partial w_{1}}\right)^{n}+\ldots
\end{aligned}
$$

Here the ellipsis denote the terms with lower conformal dimensions, which leads to less divergent terms in the limit $\epsilon \rightarrow 0 .{ }^{4}$ Under the conformal transformation (2.48), the four-point function transforms as

$$
\begin{aligned}
\langle & \left.V\left(w_{1}, \bar{w}_{1}\right) V\left(w_{1}^{\prime}, \bar{w}_{1}^{\prime}\right) V\left(w_{2}, \bar{w}_{2}\right) V\left(w_{2}^{\prime}, \bar{w}_{2}^{\prime}\right)\right\rangle \\
= & \left(\frac{\partial w_{1}}{\partial z_{1}}\right)^{h+m}\left(\frac{\partial \bar{w}_{1}}{\partial \bar{z}_{1}}\right)^{h+\bar{m}}\left(\frac{\partial w_{2}}{\partial z_{2}}\right)^{h+m}\left(\frac{\partial \bar{w}_{2}}{\partial \bar{z}_{2}}\right)^{h+\bar{m}}\left(\frac{\partial w_{1}^{\prime}}{\partial z_{1}^{\prime}}\right)^{h+m}\left(\frac{\partial \bar{w}_{1}^{\prime}}{\partial \bar{z}_{1}^{\prime}}\right)^{h+\bar{m}}\left(\frac{\partial w_{2}^{\prime}}{\partial z_{2}^{\prime}}\right)^{h+m}\left(\frac{\partial \bar{w}_{2}^{\prime}}{\partial \bar{z}_{2}^{\prime}}\right)^{h+\bar{m}} \\
& \cdot\left\langle V\left(z_{1}, \bar{z}_{1}\right) V\left(z_{1}^{\prime}, \bar{z}_{1}^{\prime}\right) V\left(z_{2}, \bar{z}_{2}\right) V\left(z_{2}^{\prime}, \bar{z}_{2}^{\prime}\right)\right\rangle+\text { less divergent terms. }
\end{aligned}
$$

Even though the operator $V(w, \bar{w})$ is not a primary operator, the coefficient for the leading term is the same as the one for the primary operator. The terms with lower conformal dimensions are less divergent in the small $\epsilon$ limit, so do not contribute to the final result.

Because we assume that the operator $V$ is a quasi-primary operator, it transforms homogeneously under a linear conformal transformation. Consider the conformal transformation

$$
u=\frac{1}{z}
$$

we have

$$
\lim _{z \rightarrow \infty} z^{2(h+m)} L^{(-)} O(z)=\lim _{z \rightarrow \infty}\left(\frac{\partial u}{\partial z}\right)^{(h+m)} z^{2(h+m)} L^{(-)} O(u)=\left.(-1)^{h+m} L^{(-)} O(u)\right|_{u=0} .
$$

Furthermore

$$
\left.L_{-n}\right|_{u=0}=\frac{1}{2 \pi i} \oint \frac{d u}{u^{n-1}} T(u)=\frac{1}{2 \pi i} \oint \frac{d z}{z^{-n+3}} T(z) z^{4}=\left.L_{n}\right|_{z \rightarrow \infty}
$$

and

$$
\lim _{z \rightarrow \infty} z^{2(h+m)}\langle 0| L^{(-)} O(z)=\langle O| L^{(-) \dagger}(-1)^{m},
$$

then the two-point function is

$$
\left\langle L^{(-)} O(z), L^{(-)} O\left(z^{\prime}\right)\right\rangle=\frac{c_{0}}{\left(z-z^{\prime}\right)^{2(h+m)}},
$$

where $c_{0}=(-1)^{m}\left\langle h\left|L^{(-) \dagger} L^{(-)}\right| h\right\rangle$.

Now let us study the divergence in the correlation function of four descendants in the $z$ coordinate. We set $l<t<l+L$ then $\left(z_{1}, z_{2}^{\prime}\right)\left(z_{2}, z_{1}^{\prime}\right)\left(\bar{z}_{1}, \bar{z}_{1}^{\prime}\right)\left(\bar{z}_{2}, \bar{z}_{2}^{\prime}\right)$ are close to each other. The four-point correlation function of quasi-primary operators can be transformed into

$$
\left\langle V\left(z_{1}, \bar{z}_{1}\right) V\left(z_{1}^{\prime}, \bar{z}_{1}^{\prime}\right) V\left(z_{2}, \bar{z}_{2}\right) V\left(z_{2}^{\prime}, \bar{z}_{2}^{\prime}\right)\right\rangle
$$

\footnotetext{
${ }^{4}$ The most divergent term in the limit $\epsilon \rightarrow 0$ comes from the OPE of two operators to the identity operator.
} 


$$
\begin{aligned}
& =\mathcal{D}\left\langle O\left(z_{1}, \bar{z}_{1}\right) O\left(z_{1}^{\prime}, \bar{z}_{1}^{\prime}\right) O\left(z_{2}, \bar{z}_{2}\right) O\left(z_{2}^{\prime}, \bar{z}_{2}^{\prime}\right)\right\rangle \\
& =\mathcal{D} \sum_{m} c_{m}\left\langle\left. O\left(z_{1}\right) O\left(z_{1}^{\prime}\right)\right|_{m} O\left(z_{2}\right) O\left(z_{2}^{\prime}\right)\right\rangle\left\langle\left. O\left(\bar{z}_{1}\right) O\left(\bar{z}_{1}^{\prime}\right)\right|_{m} O\left(\bar{z}_{2}\right) O\left(\bar{z}_{2}^{\prime}\right)\right\rangle \\
& =\mathcal{D} \sum_{m, n} c_{m, n}\left\langle\left. O\left(z_{1}\right) O\left(z_{2}^{\prime}\right)\right|_{m} O\left(z_{2}\right) O\left(z_{1}^{\prime}\right)\right\rangle\left\langle\left. O\left(\bar{z}_{1}\right) O\left(\bar{z}_{1}^{\prime}\right)\right|_{n} O\left(\bar{z}_{2}\right) O\left(\bar{z}_{2}^{\prime}\right)\right\rangle \\
& =\sum_{m, n} c_{m, n}\left\langle\left. L^{(-)} O\left(z_{1}\right) L^{(-)} O\left(z_{2}^{\prime}\right)\right|_{m} L^{(-)} O\left(z_{2}\right) L^{(-)} O\left(z_{1}^{\prime}\right)\right\rangle\left\langle\left. O\left(\bar{z}_{1}\right) O\left(\bar{z}_{1}^{\prime}\right)\right|_{n} O\left(\bar{z}_{2}\right) O\left(\bar{z}_{2}^{\prime}\right)\right\rangle,
\end{aligned}
$$

where $\left\langle\left. O\left(z_{1}\right) O\left(z_{1}^{\prime}\right)\right|_{m} O\left(z_{2}\right) O\left(z_{2}^{\prime}\right)\right\rangle$ denote the conformal block expansion with the Virasoro module $[m]$ as the propagator. The first equation transforms the correlation function of four descendants into the differential on the correlation function of corresponding primaries. In the second equation, we expand the partition function by the conformal blocks and $c_{m}$ denote the OPE coefficient. In the third equation, we expand the holomorphic part in terms of another channel [15]. In the fourth equation, we change the differential back into the Virasoro operators acting on the primaries in the correlation function. The last equation is based on the fact that the Ward identity is satisfied for the conformal blocks. The most divergent term only comes from the one with $m=n=0$ and $c_{0,0}=\frac{1}{d_{a}}$ [15]. Actually even in the vacuum block, only the identity operator gives the most divergent term,

$$
\begin{aligned}
& \left\langle V\left(z_{1}, \bar{z}_{1}\right) V\left(z_{1}^{\prime}, \bar{z}_{1}^{\prime}\right) V\left(z_{2}, \bar{z}_{2}\right) V\left(z_{2}^{\prime}, \bar{z}_{2}^{\prime}\right)\right\rangle \\
& =\frac{1}{d_{a}}\left\langle L^{(-)} O\left(z_{1}\right) L^{(-)} O\left(z_{2}^{\prime}\right)\right\rangle\left\langle L^{(-)} O\left(z_{2}\right) L^{(-)} O\left(z_{1}^{\prime}\right)\right\rangle\left\langle O\left(\bar{z}_{1}\right) O\left(\bar{z}_{1}^{\prime}\right)\right\rangle\left\langle O\left(\bar{z}_{2}\right) O\left(\bar{z}_{2}^{\prime}\right)\right\rangle \\
& \quad+\text { less divergent terms. }
\end{aligned}
$$

Changing back into the $w$-coordinate and keeping the most divergent term, we find

$$
\begin{aligned}
& \left\langle V\left(w_{1}, \bar{w}_{1}\right) V\left(w_{1}^{\prime}, \bar{w}_{1}^{\prime}\right) V\left(w_{2}, \bar{w}_{2}\right) V\left(w_{2}^{\prime}, \bar{w}_{2}^{\prime}\right)\right\rangle \\
& =\frac{1}{d_{a}}\left\langle L^{(-)} O\left(w_{1}\right) L^{(-)} O\left(w_{2}^{\prime}\right)\right\rangle\left\langle L^{(-)} O\left(w_{2}\right) L^{(-)} O\left(w_{1}^{\prime}\right)\right\rangle\left\langle O\left(\bar{w}_{1}\right) O\left(\bar{w}_{1}^{\prime}\right)\right\rangle\left\langle O\left(\bar{w}_{2}\right) O\left(\bar{w}_{2}^{\prime}\right)\right\rangle \\
& \quad+\text { less divergent terms. }
\end{aligned}
$$

Therefore, for a quasi-primary operator we still have $\Delta S_{2}=\log d_{a}$.

In short, for two kinds of descendent operators: $\partial O$ and $L^{(-)} O$, the difference between the second Rényi entropy of the excited states and the ground state is universal, equals to the logarithmic of the quantum dimension. This is the same as the case of inserting a local primary operator.

\section{$2.5 n$-th RE for generic descendent states}

With the above study, we are ready to discuss the effect of most general descendent operators. A descendent operator in a module generated from a primary operator $O_{a}$ may take the following generic form

$$
V=\sum_{m, j, r, k} d_{m, j ; r, k}\left(\partial^{m} L^{(-, j)}\right)\left(\bar{\partial}^{r} \bar{L}^{(-, k)}\right) O_{a}(w, \bar{w})
$$

where $L^{(-, j)} \bar{L}^{(-, k)} O_{a}(w, \bar{w})$ is a quasi-primary operator, and $L^{(-, j)}\left(\bar{L}^{(-, k)}\right)$ is a combination of holomorphic (anti-holomorphic) Virasoro algebra with fixed conformal dimension 


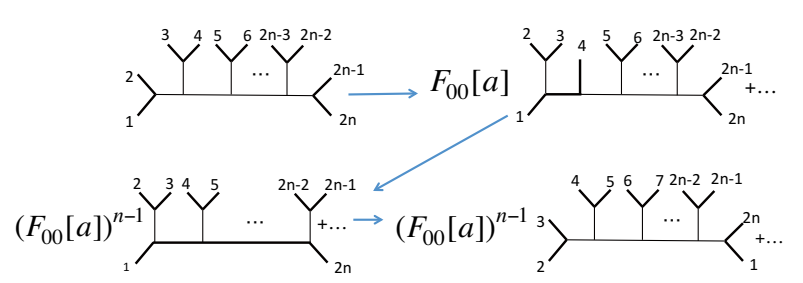

Figure 2. The fusion transformations to obtain $\Delta S_{A}^{(n)}$.

$\left[L_{0}, L^{(-, j)}\right]=p_{j} L^{(-, j)}$. Keeping the most divergent term in the correlation function, we see that only the terms with $m+r+p_{j}+\bar{p}_{k}+2 h_{a}=\Delta$ is non-zero. For general $n$-sheeted surface under the conformal transformation (2.48)

$$
\begin{aligned}
& z_{j}=\frac{(t-l-i \epsilon)^{\frac{1}{n}}}{(l+L-t+i \epsilon)^{\frac{1}{n}}} e^{\frac{2 \pi i}{n}(j-1)+\frac{\pi i}{n}}, \\
& z_{j}^{\prime}=\frac{(t-l+i \epsilon)^{\frac{1}{n}}}{(l+L-t-i \epsilon)^{\frac{1}{2}}} e^{\frac{2 \pi i}{n}(j-1)-\frac{\pi i}{n}},
\end{aligned}
$$

we see that $z_{j}$ is close to $z_{j+1}^{\prime}$ as in [15].

For the $n$-th Rényi entropy, we need to compute the $2 n$-point function. Similar to the previous calculation we need to take a conformal transformation to $z$ coordinate and take proper channel to expand the $2 n$-point function into the holomorphic and the antiholomorphic part, as graphically shown in figure $2 .{ }^{5}$ In each channel only the identity operator contribute to the final result, so the $2 n$-point function breaks up into $n$ two-point functions for the holomorphic part (and $n$ for the anti-holomorphic part). We can take a conformal transformation back to the $w$ coordinate, with the leading divergent term being transformed homogenously. Actually this recipe only needs us to know which two arguments are close to each other and the coefficients for the most divergent term. With this message we can directly do the calculation in the $w$ coordinate and multiply the coefficients from the OPE and the channel changing

$$
\begin{aligned}
& \left.\left\langle V\left(w_{1}, \bar{w}_{1}\right) V\left(w_{1}^{\prime}, \bar{w}_{1}^{\prime}\right) \ldots V\left(w_{n}, \bar{w}_{n}\right) V\left(w_{n}^{\prime}, \bar{w}_{n}^{\prime}\right)\right\rangle\right|_{n-\text { sheet }} \\
& =\sum_{m_{1}, j_{1}, r_{1}, k_{1}} \sum_{m_{1}^{\prime}, j_{1}^{\prime}, r_{1}^{\prime}, k_{1}^{\prime}} d_{m_{1}, j_{1} ; r_{1}, k_{1}} d_{m_{1}^{\prime}, j_{1}^{\prime} ; r_{1}^{\prime}, k_{1}^{\prime}}^{*} \sum_{m_{n}, j_{n}, r_{n}, k_{n}} \sum_{m_{n}^{\prime}, j_{n}^{\prime}, r_{n}^{\prime}, k_{n}^{\prime}} d_{m_{n}, j_{n} ; r_{n}, k_{n}} d_{m_{n}^{\prime}, j_{n}^{\prime} ; r_{n}^{\prime}, k_{n}^{\prime}} \\
& \left\langle\partial_{w_{1}}^{m_{1}} L^{\left(-, j_{1}\right)} \bar{\partial}_{\bar{w}_{1}}^{r_{1}} \bar{L}^{\left(-, k_{1}\right)} O_{a}\left(w_{1}, \bar{w}_{1}\right) \partial_{w_{1}^{\prime}}^{m_{1}^{\prime}} L^{\left(-, j_{1}^{\prime}\right)} \bar{\partial}_{\bar{w}_{1}}^{r_{1}^{\prime}}, \bar{L}^{\left(-, k_{1}^{\prime}\right)} O_{a}\left(w_{1}^{\prime}, \bar{w}_{1}^{\prime}\right) \ldots\right. \\
& \left.\partial_{w_{n}}^{m_{n}} L^{\left(-, j_{n}\right)} \bar{\partial}_{\bar{w}_{n}}^{r_{n}} \bar{L}^{\left(-, k_{n}\right)} O_{a}\left(w_{n}, \bar{w}_{n}\right) \partial_{w_{n}^{\prime}}^{m_{n}^{\prime}} L^{\left(-, j_{n}^{\prime}\right)} \bar{\partial}_{w_{n}^{\prime}}^{r_{n}^{\prime}}, \bar{L}^{\left(-, k_{n}^{\prime}\right)} O_{a}\left(w_{n}^{\prime}, \bar{w}_{n}^{\prime}\right)\right\rangle\left.\right|_{n-s h e e t} \\
& =d_{a}^{-(n-1)} \sum_{m_{1}, j_{1}, r_{1}, k_{1}} \sum_{m_{1}^{\prime}, j_{1}^{\prime}, r_{1}^{\prime}, k_{1}^{\prime}} d_{m_{1}, j_{1} ; r_{1}, k_{1}} d_{m_{1}^{\prime}, j_{1}^{\prime}, r_{1}^{\prime}, k_{1}^{\prime}}^{*} \sum_{m_{n}, j_{n}, r_{n}, k_{n}} \sum_{m_{n}^{\prime}, j_{n}^{\prime}, r_{n}^{\prime}, k_{n}^{\prime}} d_{m_{n}, j_{n}, r_{n}, k_{n}} d_{m_{n}^{\prime}, j_{n}^{\prime}, r_{n}^{\prime}, k_{n}^{\prime}} \\
& \left\langle\partial_{w_{1}}^{m_{1}} L^{\left(-, j_{1}\right)} O_{a}\left(w_{1}\right) \partial_{w_{2}^{\prime}}^{m_{2}^{\prime}} L^{\left(-, j_{2}^{\prime}\right)} O_{a}\left(w_{2}^{\prime}\right)\right\rangle \cdots\left\langle\partial_{w_{n}}^{m_{n}} L^{\left(-, j_{n}\right)} O_{a}\left(w_{n}\right) \partial_{w_{1}^{\prime}}^{m_{1}^{\prime}} L^{\left(-, j_{1}^{\prime}\right)} O_{a}\left(w_{1}^{\prime}\right)\right\rangle \\
& \left\langle\partial_{\bar{w}_{1}}^{r_{1}} \bar{L}^{\left(-, k_{1}\right)} O_{a}\left(\bar{w}_{1}\right) \partial_{\bar{w}_{1}^{\prime}}^{r_{1}^{\prime}} \bar{L}^{\left(-, k_{1}^{\prime}\right)} O_{a}\left(\bar{w}_{1}^{\prime}\right)\right\rangle \ldots\left\langle\partial_{\bar{w}_{n}}^{r_{n}} \bar{L}^{\left(-, k_{n}\right)} O_{a}\left(\bar{w}_{n}\right) \partial_{\bar{w}_{n}^{\prime}}^{r_{n}^{\prime}} \bar{L}^{\left(-, k_{n}^{\prime}\right)} O_{a}\left(\bar{w}_{n}^{\prime}\right)\right\rangle \\
& \text { +less divergent terms. }
\end{aligned}
$$

\footnotetext{
${ }^{5}$ We have extracted this figure from [15].
} 
Each two-point function in the above relation can be computed directly. For example, for the holomorphic part, it is

$$
\begin{aligned}
& \left\langle\partial_{w_{i}}^{m_{i}} L^{\left(-, j_{i}\right)} O_{a}\left(w_{i}\right) \partial_{w_{i+1}^{\prime}}^{m_{i+1}^{\prime}} L^{\left(-, j_{i+1}^{\prime}\right)} O_{a}\left(w_{i+1}^{\prime}\right)\right\rangle \\
& =\partial_{w_{i}}^{m_{i}} \partial_{w_{i+1}^{\prime}}^{m_{i+1}^{\prime}} \frac{\left\langle h\left|L^{\left(-, j_{i}\right) \dagger} L^{\left(-, j_{i}\right)}\right| h\right\rangle \delta_{j_{i}, j_{i+1}^{\prime}}(-1)^{p_{j_{i}}}}{\left(w_{i}-w_{i+1}^{\prime}\right)^{2\left(h+p_{j_{i}}\right)}} \\
& =\frac{\left\langle h\left|L^{\left(-, j_{i}\right) \dagger} L^{\left(-, j_{i}\right)}\right| h\right\rangle \delta_{j_{i}, j_{i+1}^{\prime}}(-1)^{p_{j_{i}}+m_{i}}}{\left(w_{i}-w_{i+1}^{\prime}\right)^{2\left(h+p_{j_{i}}\right)+m_{i}+m_{i+1}^{\prime}}}
\end{aligned}
$$

where we have used (2.70) in the first equation. Introducing the matrices

$$
\begin{aligned}
B_{\{m, j\},\{r, k\}} & =d_{m, j, r, k}^{*}, \\
M_{\{m, j\},\{r, k\}} & =\left\langle h\left|L^{(-, j) \dagger} L^{(-, j)}\right| h\right\rangle \delta_{j, k} i^{r-m},
\end{aligned}
$$

and defining the density matrix

$$
\rho=B M B^{\dagger} M^{\dagger},
$$

then the two-point and $2 n$-point function can be written as

$$
\begin{aligned}
\left\langle V\left(w_{1}, \bar{w}_{1}\right) V\left(w_{1}^{\prime}, \bar{w}_{1}^{\prime}\right)\right\rangle & =\frac{\operatorname{tr} \rho}{(2 \epsilon)^{2 \Delta}}, \\
\left.\left\langle V\left(w_{1}, \bar{w}_{1}\right) V\left(w_{1}^{\prime}, \bar{w}_{1}^{\prime}\right) \ldots V\left(w_{n}, \bar{w}_{n}\right) V\left(w_{n}^{\prime}, \bar{w}_{n}^{\prime}\right)\right\rangle\right|_{n-s h e e t} & =\frac{\operatorname{tr} \rho^{n}}{(2 \epsilon)^{2 n \Delta}} .
\end{aligned}
$$

With the normalized density matrix

$$
\rho_{0}=\frac{\rho}{\operatorname{tr} \rho}
$$

we find

$$
\frac{Z_{n}}{Z_{1}^{n}}=d_{a}^{-(n-1)} \operatorname{tr} \rho_{0}^{n}
$$

and therefore

$$
\Delta S_{n}=\log d_{a}-\frac{1}{n-1} \log \operatorname{tr} \rho_{0}^{n}=\Delta S_{n}^{\text {primary }}-\frac{1}{n-1} \log \operatorname{tr} \rho_{0}^{n},
$$

and

$$
\Delta S_{E E}=\log d_{a}-\operatorname{tr} \rho_{0} \log \rho_{0}=\Delta S_{E E}^{\text {primary }}-\operatorname{tr} \rho_{0} \log \rho_{0}
$$

where

$$
\Delta S_{n}^{\text {primary }}=\Delta S_{E E}^{\text {primary }}=\log d_{a}
$$

is the quantum entanglement of the primary operator.

It is obvious that there are two kinds of contribution to the entropy of a generic descendent operator. The first one takes a universal form, depending on the quantum dimension of the corresponding primary operator. Even though the theory could be different such that 
the OPE coefficients and the channel changing coefficients are different, the $2 n$-point function always takes the form (2.77) with a different coefficient, so the relation between the entropies of the descendant operator and the corresponding primary operator does not change.

The extra contribution to the entropy is remarkable. Formally it takes the form $-\operatorname{tr} \rho_{0} \log \rho_{0}$. It is zero only when the matrix $d_{m, j ; r, k}$ is of rank one. By matrix product, the matrix $\rho_{0}$ is also of rank one. under a conjugate transformation, $\rho_{0}$ can be diagnosed as $(1,0,0 \ldots)$, which has no more correction to the entanglement entropy and Rényi entropy. In this case the entanglement and Rényi entropy are equal to the log of quantum dimension. The two examples in the previous section $\partial O(w, \bar{w})$ and $L^{(-)} O(w, \bar{w})$ belong to this class. A general form of this kind operator is like

$$
V(w, \bar{w})=\mathcal{L}^{(-)} \overline{\mathcal{L}}^{(-)} O(w, \bar{w})
$$

where $\mathcal{L}$ and $\overline{\mathcal{L}}$ are composed of holomorphic and anti-holomorphic Virasoro generators respectively.

For the cases when the rank of matrix $d_{m, j ; r, k}$ is more than one, the entanglement entropy and Rényi entropy have extra corrections. The simplest example is for $d_{1,0 ; 0,0}=$ $d_{0,0 ; 1,0}=1$ with other coefficients being zero. This gives the operator $V=(\partial+\bar{\partial}) O(w, \bar{w})$. The entanglement entropy has extra $\log 2$ correction.

The extra increase for the entanglement entropy is easy to understand in a free theory. For the free theory, the entanglement entropy for local operator can be understood by the quasi-particle. The increase of the entanglement entropy of the local operator from the vacuum is equal to the entanglement of the EPR pair [12]. The entanglement entropy for the EPR pair only depends on the number of the pair and their relative normalizations. For the operator (2.88), we change all of the left- and right-moving particles simultaneously. Because all of the EPR pairs have the same conformal dimension their normalizations change in the same way, which do not change the entanglement entropy. However for a more generic descendant operators which can not decomposed as (2.88), it change the relative normalization of the EPR pair and even increase the number of EPR pairs. This is the origin of the extra increase in the entropies for the descendants.

From the derivation, we see that only the leading divergent term in the OPE appears in the final result. The only thing we should consider is which operators are close to each other under the analytically extension

$$
w \rightarrow-l+(t-i \epsilon), \quad \bar{w} \rightarrow-l-(t-i \epsilon) .
$$

The crucial point here is that the holomorphic and anti-holomorphic parts have different limits. For example for the four-point function $\left\langle O_{1}\left(z_{1}, \bar{z}_{1}\right) O_{2}\left(z_{2}, \bar{z}_{2}\right) O_{3}\left(z_{3}, \bar{z}_{3}\right) O_{4}\left(z_{4}, \bar{z}_{4}\right)\right\rangle$, the $\epsilon \rightarrow 0$ limit set $\left(\bar{z}_{1}, \bar{z}_{2}\right)$ close to each other while $\left(z_{1}, z_{3}\right)$ close to each other. The OPE cannot be used directly. That's the reason why we need to transform into the $z$ coordinate and use the relation between the conformal blocks in different channel.

\subsection{Comments in BCFT}

In this subsection, we would like to introduce a boundary (or defect) at $x=0$ as shown in figure 1. This boundary preserves the conformal symmetry. There are two kinds of 
boundary preserving conformal symmetry, and they correspond to the Neumann boundary condition and Dirichlet boundary condition normally. The global property of the CFT with a boundary has been discussed in $[21,29,30]$. As we know, the correlation function in a BCFT are much different from the one in CFT without boundary. In terms of (2.4), the Rényi entropy may be sensitive to the boundary. In [19], the Rényi entropy of the primary states with a boundary has been studied and it was shown that the maximal value of Rényi entropy does not change. The boundary effects just change the time evolution of Rényi entropy. It is also interesting to check what will happen to the Rényi entropy for the local descendent states in 2D CFT with a boundary. For simplicity, we only discuss the descendent operator whose entropy is the same as the one of corresponding primary operator.

As shown in $[15,19]$, the Rényi entropy highly depends on the conformal blocks of the theory for general rational CFTs in 2D. In terms of [29, 30], the $n$-point correlation functions in 2D CFTs with a boundary are related to the holomorphic part of the conformal blocks of the $2 n$-point correlation functions on the $2 \mathrm{D}$ full complex plane. There is a systematical way called the image method to translate $n$-point correlation functions in 2D BCFTs to $2 n$-point correlation functions in 2D CFT. The more precise relation is that an $n$-point function in the upper half plane(UHP), which is a function of the coordinates $\left(z_{1},, z_{n} ; \bar{z}_{1}, \ldots, \bar{z}_{n}\right)$, behaves under conformal transformations in the similar way as the holomorphic sector of an $2 n$-point function in the full plane which depends on $\left(z_{1}, \ldots, z_{n} ; z_{1}^{*}, \ldots, z_{n}^{*}\right)$, analytically continued to $z_{j}^{*}=\bar{z}_{j}$. In [15], the time evolution of Rényi entropy highly depends on the holomorphic part of conformal block. In 2D CFTs with a boundary, the boundary indeed changes the propagator of the primary field but does not change the fusion constants in the bulk. In this sense, one can expect that the time evolution of Rényi entropy of the descendent states in CFTs with a boundary is almost the same as the one in CFTs on the full complex plane. From the studies in the previous subsections, the Rényi entropy of the descendent states could be the same as the one of corresponding primary states. One can check that the boundary does not change the maximal value of the Rényi entropy of the descendent states. In terms of the quasi-particles picture given in [19], the boundary just changes the time evolution of Rényi entropy of the descendent states, just as the primary states.

\section{Rényi entropy in deformed CFT}

In the 2D rational CFT, we find that the Rényi entropy of local descendent operators could coincide with the logarithmic of the quantum dimension of the corresponding primary operator. Now we would like to study these operators in the CFTs with additional deformations or interactions. In the deformed CFTs, there is no conformal invariance. Nevertheless the effect of deformations on the Rényi entropy of local excited states can then be studied within conformal perturbation theory [31], if the deformation is weak. Here we only consider the theory which is perturbed by the local interaction $\Phi(z)$. Namely, we consider the theory which is perturbed by an operator $\Phi(z)$ with the conformal dimension $\Delta \geq 2$. The action is now

$$
I=I_{0}+\delta I=I_{0}+\lambda \int_{R_{1}} \Phi(z) d^{2} z,
$$


where $I_{0}$ is the original CFT action, $\lambda \equiv g / \Lambda^{2-\Delta}$ with $g$ being dimensionless and $R_{1}$ denotes the complex plane. Consequently the total Hamiltonian $H$ of the theory is $H=H_{0}+\delta H$, where $H_{0}$ is the Hamiltonian of original CFT. The system will be evolving under the Hamiltonian $H$ in the real time approach. As usual, we consider an excited state by acting a primary or descendent operator $O$ on the vacuum $|0\rangle$, which is defined ${ }^{6}$ by $H|0\rangle=0$.

We consider the parameter $g \ll 1$ in (3.1). The two-point correlation function of the primary or the descendent operator is

$$
\left\langle O^{\dagger}\left(\omega_{1}, \bar{\omega}_{1}\right) O\left(\omega_{2}, \bar{\omega}_{2}\right)\right\rangle_{\Sigma_{1}}=\left\langle e^{-\lambda \int_{R_{1}} \Phi(z) d^{2} z} O^{\dagger}\left(\omega_{1}, \bar{\omega}_{1}\right) O\left(\omega_{2}, \bar{\omega}_{2}\right)\right\rangle_{R_{1}},
$$

where $\Sigma_{1}$ and $R_{1}$ denote the complex plane in deformed CFT and original CFT respectively. We expand the deformation with respect to the powers of $\lambda$

$$
\left\langle e^{-\lambda \int \Phi(z) d^{2} z} \ldots\right\rangle_{R_{1}}=\sum_{N=0}^{+\infty} \frac{(-\lambda)^{N}}{N !} \int_{R_{1}} \ldots \int_{R_{1}}\left\langle\Phi\left(z_{1}\right) \ldots \Phi\left(z_{N}\right) \ldots\right\rangle_{R_{1}} d^{2} z_{1} \ldots d^{2} z_{N} .
$$

The two-point correlation function (3.2) contains infinite towers of contribution from interaction between the operators $O$ and $\Phi$ in CFT. Similarly, the 2n-point correlation function on $\Sigma_{n}$ can be expanded

$$
\begin{aligned}
& \left\langle O^{\dagger}\left(\omega_{1}, \bar{\omega}_{1}\right) O\left(\omega_{2}, \bar{\omega}_{2}\right) \ldots O\left(\omega_{2 n}, \bar{\omega}_{2 n}\right)\right\rangle_{\Sigma_{n}} \\
& =\left\langle e^{-\lambda \int \Phi(z) d^{2} z} O^{\dagger}\left(\omega_{1}, \bar{\omega}_{1}\right) O\left(\omega_{2}, \bar{\omega}_{2}\right) \ldots O\left(\omega_{2 n}, \bar{\omega}_{2 n}\right)\right\rangle_{R_{n}} \\
& =\sum_{N=0}^{+\infty} \frac{(-\lambda)^{N}}{N !} \int_{R_{n}} \ldots \int_{R_{n}}\left\langle\Phi\left(z_{1}\right) \ldots \Phi\left(z_{N}\right) O^{\dagger}\left(\omega_{1}, \bar{\omega}_{1}\right) O\left(\omega_{2}, \bar{\omega}_{2}\right) \ldots O\left(\omega_{2 n}, \bar{\omega}_{2 n}\right)\right\rangle_{R_{n}} d^{2} z_{1} \ldots d^{2} z_{N}
\end{aligned}
$$

where $\Sigma_{n}$ is $\mathrm{n}$ copies of $\Sigma_{1}$. To calculate the correlation function on $R_{n}$, one can employ the following conformal transformation

$$
\xi=\left(\frac{\omega}{\omega-l}\right)^{1 / n} \text { and } \omega=\omega(\xi)=l \frac{\xi^{n}}{\xi^{n}-1} .
$$

Then $R_{n}$ is mapped to the complex plane $R_{1}$. The points $\omega_{1}, \ldots, \omega_{2 n}$ is mapped to $z_{1}, \ldots, z_{2 n}$ respectively by (3.4). The $2 n$-point correlation function can be expressed as

$$
\begin{aligned}
& \left\langle O^{\dagger}\left(\omega_{1}, \bar{\omega}_{1}\right) O\left(\omega_{2}, \bar{\omega}_{2}\right) \ldots O\left(\omega_{2 n}, \bar{\omega}_{2 n}\right)\right\rangle_{\Sigma_{n}} \\
& =\sum_{N=0}^{+\infty} C_{n} \frac{(-\lambda)^{N}}{N !} \int_{R_{1}} \ldots \int_{R_{1}}\left(\omega^{\prime}\left(\tilde{\xi}_{1}\right) \ldots \omega^{\prime}\left(\tilde{\xi}_{N}\right)\right)^{2-\Delta}\left\langle\Phi\left(\tilde{\xi}_{1}\right) \ldots \Phi\left(\tilde{\xi}_{N}\right)\right. \\
& \left.\quad \times O^{\dagger}\left(\xi_{1}, \bar{\xi}_{1}\right) O\left(\xi_{2}, \bar{\xi}_{2}\right) \ldots O\left(\xi_{2 n}, \bar{\xi}_{2 n}\right)\right\rangle_{R_{1}} d^{2} \tilde{\xi}_{1} \ldots d^{2} \tilde{\xi}_{N},
\end{aligned}
$$

where $C_{n}$ is the Jacobian from $O\left(\omega_{1}\right) \ldots O\left(\omega_{n}\right)$. We know that when $L+l<t$ or $t<L$, $\xi_{i}-\xi_{i+1} \sim O(\epsilon)(i=1, \ldots, 2 n-1)$. Then the leading order term in the $2 n$-point correlation function is

$$
\left\langle O^{\dagger}\left(\omega_{1}, \bar{\omega}_{1}\right) O\left(\omega_{2}, \bar{\omega}_{2}\right) \ldots O\left(\omega_{2 n}, \bar{\omega}_{2 n}\right)\right\rangle_{\Sigma_{n}}
$$

\footnotetext{
${ }^{6}$ We set the vacuum energy to be zero for convenience.
} 


$$
\begin{aligned}
\simeq & \sum_{N=0}^{+\infty} C_{n} \frac{(-\lambda)^{N}}{N !} \int_{R_{1}} \ldots \int_{R_{1}}\left(\omega^{\prime}\left(\tilde{\xi}_{1}\right) \ldots \omega^{\prime}\left(\tilde{\xi}_{N}\right)\right)^{2-\Delta}\left\langle\Phi\left(\tilde{\xi}_{1}\right) \ldots \Phi\left(\tilde{\xi}_{N}\right)\right\rangle_{R_{1}} d^{2} \tilde{\xi}_{1} \ldots d^{2} \tilde{\xi}_{N} \\
& \times \prod_{i=1}^{n-1}\left\langle O^{\dagger}\left(\xi_{2 i+1}, \bar{\xi}_{2 i+1}\right) O\left(\xi_{2 i+2}, \bar{\xi}_{2 i+2}\right)\right\rangle_{R_{1}} .
\end{aligned}
$$

In (3.6), the contribution from $O\left(z_{i}\right)$ being contracted with $\Phi\left(\bar{\xi}_{1}\right)$ is subleading in the limit $\epsilon \rightarrow 0$. There are some subtle issues which have been come across in perturbative CFT. It is well known that such integrals are potentially ambiguous due to the singularities from the contact terms when $O\left(z_{i}\right)$ contracts with $\Phi\left(\bar{\xi}_{j}\right)[32,33]$. After proper regularization which highly depends on the specific choice of $O$ and $\Phi$, the contraction between $O$ and $\Phi$ make a finite contribution. That means the dominant contribution in the limit $\epsilon \rightarrow 0$ comes only from the contraction between $O$ 's as shown in the last line of (3.6). In order to present the crucial point clearly, we take the second Rényi entropy as an example. The second Rényi entropy (2.4) can be expanded by the powers of $\lambda$ as following

$$
\begin{aligned}
\Delta S_{A}^{(2)}=\log [ & \frac{\langle O O O O\rangle_{R_{2}}}{\langle O O\rangle_{R_{1}}^{2}}-2 \lambda \frac{\langle O O O O\rangle_{R_{2}}}{\langle O O\rangle_{R_{1}}} \int\langle\Phi(\tilde{\xi}) O O\rangle_{R_{1}}\left(\omega^{\prime}(\tilde{\xi})\right)^{2-\Delta} d \tilde{\xi} \\
& \left.+\frac{\lambda C_{2}}{\langle O O\rangle_{R_{1}}^{2}} \int d \tilde{\xi}\left(\omega^{\prime}(\tilde{\xi})\right)^{2-\Delta}\langle\Phi(\tilde{\xi}) O O O O\rangle_{R_{1}}+\ldots\right],
\end{aligned}
$$

where the ... stands for the higher order terms in $g$. In order to make the perturbation well defined, we need a regularization to deal with the divergent terms in the integration. Especially for $\int_{R_{1}}\left(\omega^{\prime}(\tilde{\xi})\right)^{2-\Delta}\left\langle\Phi(\tilde{\xi}) O\left(w_{1}\right) O\left(w_{2}\right)\right\rangle_{R_{1}} d \tilde{\xi}$, we should introduce two contract terms like $\int\left(\omega^{\prime}(\tilde{\xi})\right)^{2-\Delta}\left\langle\Phi(\tilde{\xi}) O\left(w_{1}\right) O\left(w_{2}\right)\right\rangle_{R_{1}}\left(\delta\left(\tilde{\xi}-w_{1}\right)+\delta\left(\tilde{\xi}-w_{2}\right)\right) d \tilde{\xi}$ to subtract the divergence if $\Phi$ is close to $O$. For the second integration in (3.7), we should do a similar regularization to deal with the divergence. All the divergent terms can be absorbed into $\lambda$ to make the perturbation well defined. Finally, $\left\langle\Phi O\left(z_{1}\right) O\left(z_{2}\right) O\left(z_{3}\right) O\left(z_{4}\right)\right\rangle_{R_{2}}$ can be expressed by $\langle\Phi\rangle\left\langle O\left(z_{1}\right) O\left(z_{2}\right) O\left(z_{3}\right) O\left(z_{4}\right)\right\rangle_{R_{1}}$ in the early time limit or the late time limit (2.11). Repeating the analysis, we can calculate the $\Delta S_{A}^{(2)}$ to higher order of $\lambda$ formally. For example,

$$
\begin{aligned}
\Delta S_{A}^{(2)}=\log [ & \frac{\langle O O O O\rangle_{R_{2}}}{\langle O O\rangle_{R_{1}}^{2}}-2 \lambda C_{2}\langle O O O O\rangle_{R_{2}} \int\langle\Phi(\tilde{\xi})\rangle_{R_{1}}\left(\omega^{\prime}(\tilde{\xi})\right)^{2-\Delta} d \tilde{\xi} \\
& \left.+\frac{\lambda C_{2}\langle O O O O\rangle_{R_{2}}}{\langle O O\rangle_{R_{1}}^{2}} \int d \tilde{\xi}\left(\omega^{\prime}(\tilde{\xi})\right)^{2-\Delta}\langle\Phi(\tilde{\xi})\rangle_{R_{1}}+\ldots\right] .
\end{aligned}
$$

In the relation (3.8), $\frac{\langle O O O O\rangle_{R_{2}}}{\langle O O\rangle_{R_{1}}^{2}}=1 / d_{O}$ which corresponds to the contribution from the local excitation during the time $l<t<L+l$.

If $\Phi$ is a primary field, $\langle\Phi\rangle_{R_{1}}=0$. We consider the second order of $\lambda$. The two-point function is

$$
\left\langle O^{\dagger}\left(\omega_{1}, \bar{\omega}_{1}\right) O\left(\omega_{2}, \bar{\omega}_{2}\right)_{\Sigma_{1}}=\left\langle O^{\dagger}\left(\omega_{1}, \bar{\omega}_{1}\right) O\left(\omega_{2}, \bar{\omega}_{2}\right)_{R_{1}}\left(1+\frac{1}{2} \lambda^{2} \int_{R_{1}}\left\langle\Phi\left(z_{1}\right) \Phi\left(z_{2}\right)\right\rangle_{R_{1}} d^{2} z_{1} d^{2} z_{2}\right) .\right.\right.
$$

Similarly, the $2 n$-point function

$$
\left\langle O^{\dagger}\left(\omega_{1}, \bar{\omega}_{1}\right) O\left(\omega_{2}, \bar{\omega}_{2}\right) \ldots O\left(\omega_{2 n}, \bar{\omega}_{2 n}\right)\right\rangle_{\Sigma_{n}}
$$




$$
\simeq C_{n} \prod_{i=1}^{n-1}\left\langle O^{\dagger}\left(\xi_{2 i+1}, \bar{\xi}_{2 i+1}\right) O\left(\xi_{2 i+2}, \bar{\xi}_{2 i+2}\right)\right\rangle\left(1+\frac{1}{2} \lambda^{2} \int_{R_{n}}\left\langle\Phi\left(z_{1}\right) \Phi\left(z_{2}\right)\right\rangle_{R_{n}} d^{2} z_{1} d^{2} z_{2}\right),
$$

during the time $L+l<t$ or $0<t<l$. Then

$$
\begin{aligned}
\Delta S^{(n)} & =\Delta S_{C F T}^{(n)}+\frac{1}{1-n} \log \frac{1+\frac{1}{2} \lambda^{2} \int\left\langle\Phi\left(z_{1}\right) \Phi\left(z_{2}\right)\right\rangle_{R_{n}} d^{2} z_{1} d^{2} z_{2}}{\left(1+\frac{1}{2} \lambda^{2} \int\left\langle\Phi\left(z_{1}\right) \Phi\left(z_{2}\right)\right\rangle_{R_{1}} d^{2} z_{1} d^{2} z_{2}\right)^{n}} \\
& \simeq \Delta S_{C F T}^{(n)}+\frac{1}{2(1-n)} \lambda^{2}\left(\int\left\langle\Phi\left(z_{1}\right) \Phi\left(z_{2}\right)\right\rangle_{R_{n}} d^{2} z_{1} d^{2} z_{2}-n \int\left\langle\Phi\left(z_{1}\right) \Phi\left(z_{2}\right)\right\rangle_{R_{1}} d^{2} z_{1} d^{2} z_{2}\right),
\end{aligned}
$$

where $\Delta S_{C F T}^{(n)}$ denotes the result in the CFT. The second part in (3.10) is just the correction to the Rényi entropy from the deformation. It is called global contribution in the paper [31]. During the time $l<t<L+l$ the only change is in $\Delta S_{C F T}^{(n)}$, which contributes the $\log d_{O}$.

\section{Conclusion and discussion}

In this paper, we have studied the Rényi entropy of local descendent operators in 2D CFT, extending the previous studies in $[12,15,19]$. In $[12,15,19]$, it has been found that the Rényi entropy of a primary state is equal to the logarithmic of quantum dimension of the primary operator. It is a natural question to consider the quantum entanglement of the descendent states. Firstly, we showed that for the specific operator $L_{-1} O$ with $O$ being primary, its Rényi entropy is still the logarithmic of quantum dimension of the primary operator $O$. Secondly, we discuss the descendent state $L^{(-)} O|0\rangle$ generated by a quasi-primary operator. For such quasi-primary states, we showed that their quantum entanglements are the same as their primaries. Despite the fact that the operators look quite complicated and their conformal transformations are involved, the leading divergent terms in the early time and late time limit are simple, behaving as the one for primary operators. As a result, the quantum entanglement of the quasi-primary operators are the same as the primaries. Moreover we discussed the most generic descendent operators of the form

$$
V=\sum_{m, j, r, k} d_{m, j ; r, k}\left(\partial^{m} L^{(-, j)}\right)\left(\bar{\partial}^{r} \bar{L}^{(-, k)}\right) O_{a}(w, \bar{w}) .
$$

Out of surprise, we found that the Rényi entropy of such operator is generally different from the one of the primary $O_{a}(w, \bar{w})$. Only when the rank of the matrix $d_{m, j ; r, k}$ is one, the entropies are the same as the ones of the primary. A typical example of such operator is

$$
V^{(1)}(w, \bar{w})=\mathcal{L}^{(-)} \overline{\mathcal{L}}^{(-)} \mathcal{O}
$$

Otherwise there is extra contribution. A typical example with extra contribution is the operator of the form

$$
V^{(2)}(w, \bar{w})=\left(L^{-}+\bar{L}^{-}\right) O(w, \bar{w}) .
$$

To clarify the entropy difference between two kinds of operators $V^{(1)}(w, \bar{w})$ and $V^{(2)}(w, \bar{w})$, it would be illuminating to consider the free scalar field theory $\phi$ in $2 \mathrm{D}$. Consider the primary operators $O_{1}$ and $O_{2}$, with

$$
O_{1}=e^{i \phi}, \quad O_{2}=\frac{1}{\sqrt{2}}\left(e^{i \phi}+e^{-i \phi}\right),
$$


where $\phi \equiv \phi(z)+\bar{\phi}(\bar{z})$. Following $[12,15]$ the excited state $O_{1}|0\rangle$ is regarded as the product state $e^{i \phi(z)}|0\rangle_{L} \otimes e^{i \phi(\bar{z})}|0\rangle_{R}$ in the chiral and anti-chiral sectors. It is not an entangled state, so we get a vanishing entanglement entropy. On the other hand, the operator $\mathrm{O}_{2}$ creates the maximally entangled state, $\frac{1}{\sqrt{2}}\left(e^{i \phi(z)}|0\rangle_{L} \otimes e^{i \bar{\phi}(\bar{z})}|0\rangle_{R}+e^{-i \phi(z)}|0\rangle_{L} \otimes e^{-i \bar{\phi}(\bar{z})}|0\rangle_{R}\right)$. The Rényi entropy is $\log 2$ when one of the sector spreads into the region of the subsystem. The first descendent operators $O_{1}^{(-1)}=L_{-1} O_{1}$ and $O_{2}^{(-1)}=L_{-1} O_{2}$ are

$$
O_{1}^{(-1)}=i \partial \phi(z) e^{i \phi}, \quad O_{2}^{(-1)}=\frac{i}{\sqrt{2}} \partial \phi(z)\left(e^{i \phi}+e^{-i \phi}\right) .
$$

The state $O_{1}^{(-1)}|0\rangle=\left(i \partial \phi(z) e^{i \phi(z)}\right)|0\rangle_{L} \otimes e^{i \bar{\phi}(\bar{z})}|0\rangle_{R}$ is still a product state, has vanishing the Rényi entropy. Similarly, the state $O_{2}^{(-1)}|0\rangle=\frac{1}{\sqrt{2}}\left[\left(i \partial \phi(z) e^{i \phi(z)}\right)|0\rangle_{L} \otimes e^{i \bar{\phi}(\bar{z})}|0\rangle_{R}-\right.$ $\left.\left(i \partial \phi(z) e^{-i \phi(z)}\right)|0\rangle_{L} \otimes e^{-i \bar{\phi}(\bar{z})}|0\rangle_{R}\right]$ is still a maximally entangled state. Therefore, the Rényi entropy should be same as the original state. We could do the operation on the chiral sector by $\prod_{i} L_{-i}^{k_{i}}\left(i, k_{i}=1,2, \ldots\right)$ any times. The result is just a new EPR state. The operation by $L_{-i}$ does not change the entanglement property, but just change the state in the chiral sector. This is the basic reason that the local excitation by the descendent operators works similarly as the primary operator in this class. The same argument could also be used for the operations $\prod_{i} \bar{L}_{-i}^{k_{i}}\left(i, k_{i}=1,2, \ldots\right)$.

Now Let us see what will happen when we apply the operation $\sum_{\left\{n_{i}\right\}\left\{n_{j}\right\}}\left(\prod_{i} L_{-n_{i}}+\right.$ $\left.\prod_{j} \bar{L}_{-n_{j}}\right)$, e.g., $L_{-1}+\bar{L}_{-1}$. The operators $O_{1}$ and $O_{2}$ become $^{7}$

$$
\begin{aligned}
& O_{1}^{(-1,-\overline{1})} \equiv\left(L_{-1}+\bar{L}_{-1}\right) O_{1}=(i \partial \phi(z)+i \bar{\partial} \bar{\phi}(\bar{z})) e^{i \phi(z)+i \bar{\phi}(\bar{z})}, \\
& O_{2}^{(-1,-\overline{1})} \equiv\left(L_{-1}+\bar{L}_{-1}\right) O_{2}=(i \partial \phi(z)+i \bar{\partial} \bar{\phi}(\bar{z}))\left(e^{i \phi}-e^{-i \phi}\right) .
\end{aligned}
$$

For the operator $O_{1}^{(-1,-\overline{1})}$, the corresponding state is

$$
O_{1}^{(-1,-\overline{1})}|0\rangle=|2\rangle_{L} \otimes|1\rangle_{R}+|1\rangle_{L} \otimes|2\rangle_{R}
$$

where we define

$$
|1\rangle_{L}=e^{i \phi(z)}|0\rangle_{L},|2\rangle_{L}=i \partial \phi(z) e^{i \phi(z)}|0\rangle_{L},|1\rangle_{R}=e^{i \bar{\phi}(\bar{z})}|0\rangle_{R},|2\rangle_{R}=i \bar{\partial} \bar{\phi}(\bar{z}) e^{i \bar{\phi}(\bar{z})}|0\rangle_{R} .
$$

With some normalization this is just the EPR state, the entanglement entropy of which is $\log 2$ when one of the sector state spreads into the subsystem $A$. In our calculation this contribution comes from the second term in (2.86). For the operator $O_{2}^{(-1,-\overline{1})}$, the state is

$$
O_{2}^{(-1,-\overline{1})}|0\rangle=\left(|2\rangle_{L}|1\rangle_{R}+|1\rangle_{L}|2\rangle_{R}\right)-\left(|4\rangle_{L}|3\rangle_{R}+|3\rangle_{L}|4\rangle_{R}\right)
$$

where we define

$$
|3\rangle_{L}=e^{-i \phi(z)}|0\rangle_{L},|4\rangle_{L}=i \partial \phi(z) e^{-i \partial \phi(z)}|0\rangle_{L},|3\rangle_{R}=e^{-i \bar{\phi}(\bar{z})}|0\rangle_{R},|4\rangle_{R}=i \bar{\partial} \bar{\phi}(\bar{z}) e^{-i \bar{\partial} \bar{\phi}(\bar{z})}|0\rangle_{R} .
$$

We could see that after normalization the relation (4.8) is just the direct sum of two EPR states. The entanglement entropy is $2 \log 2$ when one of the sector state spreads into the subsystem $A$. This is also consistent with our calculation (2.86).

\footnotetext{
${ }^{7}$ We ignore the constant $\frac{1}{\sqrt{2}}$ for $O_{2}$ below.
} 
Indeed for the interacting theory it is not easy to find such a clear explanation. Actually we still need better understanding on the fact that the seemingly decoupled chiral and antichiral sector are entangled with each other, even for the primary excitation state, see [39].

In this paper, we also introduced a boundary which do not break conformal symmetry as in [19]. For the descendent state of type (1.1), we estimated the maximal value of Rényi entropy of local descendent states, which is the same as the one in the theory without such kind of boundary, although the boundary change the time evolution behavior of Rényi entropy. Finally, we deformed the original CFT by additional operator and discussed the Rényi entropy of generic local excited states. If the deformation is small, we can treat it as a perturbation of CFT. By this way, we can estimate that this deformation make a global contribution to the Rényi entropy up to $g^{2}$, if the deformation is a primary field. Though there are divergences in the global contribution [31], such divergences can be regulated appropriately. Actually there are various definite extensive examples [34-38] to show that global contribution is finite.

\section{Acknowledgments}

We are grateful to J. L. Cardy, Mitsutoshi Fujita, Rene Meyer, Masahiro Nozaki, T. Numasawa, Tadashi Takayanagi and K. Watanabe for useful conversations and correspondence. W.G. and S.H. thank Miao Li, Tadashi Takayanagi for their encouragement and support. S. H would like to thank to Sichuan University, IPMU, and Max Planck Institute for Gravitational Physics (AEI) for hospitality. BC and J.q. Wu was supported in part by NSFC Grants No. 11275010, No. 11335012 and No. 11325522. W. Z. Guo is supported by Postgraduate Scholarship Program of China Scholarship Council. S.H. is supported by JSPS postdoctoral fellowship for foreign researchers and by the National Natural Science Foundation of China (No.11305235).

\section{A Conformal transformation for descendent operator}

In this section, we show how to take the conformal transformation for a decedent operator. For a general primary operator, under a conformal transformation it acts as

$$
\psi(z)=\psi(w)\left(\frac{\partial w}{\partial z}\right)^{h}\left(\frac{\partial \bar{w}}{\partial \bar{z}}\right)^{\bar{h}} .
$$

First consider the conformal transformation of $L_{-1} \psi(z)$

$$
\begin{aligned}
L_{-1} \psi(z) & =\frac{1}{2 \pi i} \oint d z^{\prime} z^{\prime n+1} T\left(z^{\prime}\right) \psi(z) \\
& =\frac{1}{2 \pi i} \oint d z^{\prime}\left[T\left(w^{\prime}\right)\left[\frac{\partial w^{\prime}}{\partial z^{\prime}}+\frac{c}{12}\left\{w^{\prime}, z^{\prime}\right\}\right] \psi(w)\left(\frac{\partial w}{\partial z}\right)^{h}\left(\frac{\partial \bar{w}}{\partial \bar{z}}\right)^{\bar{h}}\right. \\
& =\frac{1}{2 \pi i} \oint d z^{\prime}\left[T\left(w^{\prime}\right)\left(\frac{\partial w^{\prime}}{\partial z^{\prime}}\right)^{2}\right] \psi(w)\left(\frac{\partial w}{\partial z}\right)^{h}\left(\frac{\partial \bar{w}}{\partial \bar{z}}\right)^{h} \\
& =\frac{1}{2 \pi i} \oint d z^{\prime}\left(\frac{\partial w^{\prime}}{\partial z^{\prime}}\right)^{2}\left[\frac{h \psi(w)}{\left(w^{\prime}-w\right)^{2}}+\frac{\partial_{w} \psi(w)}{w^{\prime}-w}+\text { normal }\right]\left(\frac{\partial w}{\partial z}\right)^{h}\left(\frac{\partial \bar{w}}{\partial \bar{z}}\right)^{\bar{h}}
\end{aligned}
$$




$$
=\left(\frac{\frac{\partial^{2} w}{\partial z^{2}}}{\frac{\partial w}{\partial z}} h \psi(w)+\left(\frac{\partial w}{\partial z}\right) \partial_{w} \psi(w)\right)\left(\frac{\partial w}{\partial z}\right)^{h}\left(\frac{\partial \bar{w}}{\partial \bar{z}}\right)^{\bar{h}}
$$

This is really the conformal transformation for the operator $\partial \psi(z)$, which support our calculation.

For more generic descendent operator

$$
\begin{aligned}
L_{-n} \psi(z)= & \frac{1}{2 \pi i} \oint d z^{\prime}\left(z^{\prime}-z\right)^{-n+1} T\left(z^{\prime}\right) \psi(z) \\
= & \frac{1}{2 \pi i} \oint d z^{\prime}\left(z^{\prime}-z\right)^{-n+1}\left[T\left(w^{\prime}\right)\left(\frac{\partial w^{\prime}}{\partial z^{\prime}}\right)^{2}+\frac{c}{12}\left\{w^{\prime}, z^{\prime}\right\}\right] \psi(w)\left(\frac{\partial w}{\partial z}\right)^{h}\left(\frac{\partial \bar{w}}{\partial \bar{z}}\right)^{\bar{h}} \\
= & \frac{1}{2 \pi i} \oint d z^{\prime}\left(z^{\prime}-z\right)^{-n+1}\left[\left(\frac{\partial w^{\prime}}{\partial z^{\prime}}\right)^{2}\left(\frac{h \psi(w)}{\left(w^{\prime}-w\right)^{2}}+\frac{\partial_{w} \psi(w)}{w^{\prime}-w}+: T\left(w^{\prime}\right) \psi(w):\right)\right. \\
& \left.+\frac{c}{12}\left\{w^{\prime}, z^{\prime}\right\} \psi(w)\right]\left(\frac{\partial w}{\partial z}\right)^{h}\left(\frac{\partial \bar{w}}{\partial \bar{z}}\right)^{\bar{h}}
\end{aligned}
$$

Calculating the contour integral, the conformal transformation of specific descendent operator is

$$
\begin{aligned}
L_{-2} \psi(z)= & \left(-\frac{1}{4}\left(\frac{\frac{\partial^{2} w}{\partial z^{2}}}{\frac{\partial w}{\partial z}}\right)^{2}+\frac{2}{3} \frac{\frac{\partial^{3} w}{\partial z^{3}}}{\frac{\partial w}{\partial z}}\right) h \psi(w)\left(\frac{\partial w}{\partial z}\right)^{h}\left(\frac{\partial \bar{w}}{\partial \bar{z}}\right)^{\bar{h}} \\
& +\frac{3}{2} \frac{\partial^{2} w}{\partial z^{2}} \partial_{w} \psi(w)\left(\frac{\partial w}{\partial z}\right)^{h}\left(\frac{\partial \bar{w}}{\partial \bar{z}}\right)^{\bar{h}}+\left(\frac{\partial w}{\partial z}\right)^{2} L_{-2} \psi(w)\left(\frac{\partial w}{\partial z}\right)^{h}\left(\frac{\partial \bar{w}}{\partial \bar{z}}\right)^{\bar{h}} \\
& +\frac{c}{12}\{w, z\}\left(\frac{\partial w}{\partial z}\right)^{2} \psi(w)\left(\frac{\partial w}{\partial z}\right)^{h}\left(\frac{\partial \bar{w}}{\partial \bar{z}}\right)^{\bar{h}}
\end{aligned}
$$

Actually, we can get a more general conformal transformation for the operators, which is an expansion for the previous result

$$
\begin{aligned}
\left.L_{-2}\right|_{z_{1}}= & \frac{1}{2 \pi i} \oint d z \frac{1}{z-z_{1}} T(z) \\
= & \frac{1}{2 \pi i} \oint d z \frac{1}{z-z_{1}}\left(T(w)\left(\frac{\partial w}{\partial z}\right)^{2}+\frac{c}{12}\{w, z\}\right) \\
= & \frac{1}{2 \pi i} \oint d z \frac{1}{z-z_{1}}\left(\left(w-w_{1}\right) L_{-3}+L_{-2}+\frac{L_{-1}}{w-w_{1}}+\frac{L_{0}}{\left(w-w_{1}\right)^{2}}+\frac{L_{1}}{\left(w-w_{1}\right)^{3}}+\frac{L_{2}}{\left(w-w_{1}\right)^{4}}\right. \\
& + \text { higher order })\left(\frac{\partial w}{\partial z}\right)^{2}+\frac{c}{12}\left\{w_{1}, z_{1}\right\} \\
= & L_{-2}\left(\frac{\partial w_{1}}{\partial z_{1}}\right)^{2}+L_{-1} \frac{3}{2} \frac{\partial^{2} w_{1}}{\partial z_{1}^{2}}+L_{0}\left(-\frac{1}{4}\left(\frac{\frac{\partial^{2} w_{1}}{\partial z_{1}^{2}}}{\frac{\partial w_{1}}{\partial z_{1}}}\right)^{2}+\frac{2}{3} \frac{\frac{\partial^{3} w_{1}}{\partial z_{1}^{3}}}{\frac{\partial w_{1}}{\partial z_{1}}}\right) \\
& +L_{1} \frac{1}{\frac{\partial w_{1}}{\partial z_{1}}} \frac{1}{24}\left(6\left(\frac{\frac{\partial^{2} w_{1}}{\partial z_{1}^{2}}}{\frac{\partial w_{1}}{\partial z_{1}}}\right)^{3}-12 \frac{\frac{\partial^{2} w_{1}}{\partial z_{1}^{2}}}{\frac{\partial^{3} w_{1}}{\partial z_{1}^{3}}}+5 \frac{\frac{\partial^{4} w_{1}}{\partial z_{1}^{4}}}{\frac{\partial w_{1}}{\partial z_{1}}}\right)^{2} \\
& +L_{2} \frac{1}{\left(\frac{\partial w_{1}}{\partial z_{1}}\right)^{2}} \frac{1}{720}\left[-225\left(\frac{\frac{\partial^{2} w_{1}}{\partial z_{1}^{2}}}{\frac{\partial w_{1}}{\partial z_{1}}}\right)^{4}+480\left(\frac{\frac{\partial^{2} w_{1}}{\partial z_{1}^{2}}}{\frac{\partial w_{1}}{\partial z_{1}}}\right)^{2} \frac{\partial^{3} w_{1}}{\frac{\partial z_{1}^{3}}{\frac{\partial w_{1}}{\partial z_{1}}}-100\left(\frac{\frac{\partial^{3} w_{1}}{\partial z_{1}^{3}}}{\frac{\partial w_{1}}{\partial z_{1}}}\right)^{2}-180 \frac{\frac{\partial^{2} w_{1}}{\partial z_{1}^{2}}}{\frac{\partial w_{1}}{\partial z_{1}}} \frac{\frac{\partial^{4} w_{1}}{\partial z_{1}^{4}}}{\frac{\partial w_{1}}{\partial z_{1}}}}\right.
\end{aligned}
$$




$$
\left.+36 \frac{\frac{\partial^{5} w_{1}}{\partial z_{1}^{5}}}{\frac{\partial w_{1}}{\partial z_{1}}}\right]+ \text { higher orders }
$$

Open Access. This article is distributed under the terms of the Creative Commons Attribution License (CC-BY 4.0), which permits any use, distribution and reproduction in any medium, provided the original author(s) and source are credited.

\section{References}

[1] A. Kitaev and J. Preskill, Topological entanglement entropy, Phys. Rev. Lett. 96 (2006) 110404 [hep-th/0510092] [INSPIRE].

[2] M. Levin and X.G. Wen, Detecting topological order in a ground state wave function, Phys. Rev. Lett. 96 (2006) 110405 [cond-mat/0510613].

[3] P. Fendley, M.P.A. Fisher and C. Nayak, Topological entanglement entropy from the holographic partition function, J. Statist. Phys. 126 (2007) 1111 [cond-mat/0609072] [INSPIRE].

[4] P. Calabrese and J.L. Cardy, Entanglement entropy and quantum field theory, J. Stat. Mech. 06 (2004) P06002 [hep-th/0405152] [InSPIRE].

[5] G.W. Moore and N. Seiberg, Polynomial equations for rational conformal field theories, Phys. Lett. B 212 (1988) 451 [INSPIRE].

[6] G.W. Moore and N. Seiberg, Classical and quantum conformal field theory, Commun. Math. Phys. 123 (1989) 177 [INSPIRE].

[7] E.P. Verlinde, Fusion rules and modular transformations in $2 D$ conformal field theory, Nucl. Phys. B 300 (1988) 360 [INSPIRE].

[8] J.L. Cardy, Boundary conditions, fusion rules and the Verlinde formula, Nucl. Phys. B 324 (1989) 581 [INSPIRE].

[9] R. Dijkgraaf and E.P. Verlinde, Modular invariance and the fusion algebra, Nucl. Phys. Proc. Suppl. B 5 (1988) 87 [INSPIRE].

[10] F.C. Alcaraz, M.I. Berganza and G. Sierra, Entanglement of low-energy excitations in conformal field theory, Phys. Rev. Lett. 106 (2011) 201601 [arXiv:1101.2881] [InSPIRE].

[11] T. Pálmai, Excited state entanglement in one dimensional quantum critical systems: extensivity and the role of microscopic details, Phys. Rev. B 90 (2014) 161404 [arXiv: 1406.3182] [INSPIRE].

[12] M. Nozaki, T. Numasawa and T. Takayanagi, Quantum entanglement of local operators in conformal field theories, Phys. Rev. Lett. 112 (2014) 111602 [arXiv:1401.0539] [INSPIRE].

[13] M. Nozaki, Notes on quantum entanglement of local operators, JHEP 10 (2014) 147 [arXiv: 1405.5875] [INSPIRE].

[14] N. Shiba, Entanglement entropy of disjoint regions in excited states: an operator method, JHEP 12 (2014) 152 [arXiv: 1408.0637] [INSPIRE].

[15] S. He, T. Numasawa, T. Takayanagi and K. Watanabe, Quantum dimension as entanglement entropy in two dimensional conformal field theories, Phys. Rev. D 90 (2014) 041701 [arXiv:1403.0702] [INSPIRE]. 
[16] P. Caputa, M. Nozaki and T. Takayanagi, Entanglement of local operators in large- $N$ conformal field theories, Prog. Theor. Exp. Phys. 2014 (2014) 093B06 [arXiv:1405.5946] [INSPIRE].

[17] C.T. Asplund, A. Bernamonti, F. Galli and T. Hartman, Holographic entanglement entropy from 2d CFT: heavy states and local quenches, JHEP 02 (2015) 171 [arXiv:1410.1392] [INSPIRE].

[18] P. Caputa, J. Simón, A. Štikonas and T. Takayanagi, Quantum entanglement of localized excited states at finite temperature, JHEP 01 (2015) 102 [arXiv:1410.2287] [INSPIRE].

[19] W.-Z. Guo and S. He, Rényi entropy of locally excited states with thermal and boundary effect in 2D CFTs, JHEP 04 (2015) 099 [arXiv:1501.00757] [INSPIRE].

[20] D. Das and S. Datta, Universal features of left-right entanglement entropy, Phys. Rev. Lett. 115 (2015) 131602 [arXiv:1504.02475] [INSPIRE].

[21] P. Calabrese and J.L. Cardy, Evolution of entanglement entropy in one-dimensional systems, J. Stat. Mech. 04 (2005) P04010 [cond-mat/0503393] [INSPIRE].

[22] V.S. Dotsenko and V.A. Fateev, Conformal algebra and multipoint correlation functions in two-dimensional statistical models, Nucl. Phys. B 240 (1984) 312 [INSPIRE].

[23] V.S. Dotsenko and V.A. Fateev, Four point correlation functions and the operator algebra in the two-dimensional conformal invariant theories with the central charge $c<1$, Nucl. Phys. B 251 (1985) 691 [INSPIRE].

[24] Ph. Di Francesco, P. Mathieu and D. Sénéchal, Conformal field theory, Springer, Germany (1998).

[25] A.A. Belavin, A.M. Polyakov and A.B. Zamolodchikov, Infinite conformal symmetry in two-dimensional quantum field theory, Nucl. Phys. B 241 (1984) 333 [InSPIRE].

[26] S. Ferrara, A.F. Grillo and R. Gatto, Tensor representations of conformal algebra and conformally covariant operator product expansion, Annals Phys. 76 (1973) 161 [INSPIRE].

[27] A.M. Polyakov, Non-Hamiltonian approach to the quantum field theory at small distances, submitted to Zh. Eksp. Teor. Fiz. (1974) [INSPIRE].

[28] H. Osborn, Conformal blocks for arbitrary spins in two dimensions, Phys. Lett. B 718 (2014) 169 [arXiv:1205.1941] [INSPIRE].

[29] J.L. Cardy, Conformal invariance and surface critical behavior, Nucl. Phys. B 240 (1984) 514 [INSPIRE].

[30] J.L. Cardy, Boundary conditions, fusion rules and the Verlinde formula, Nucl. Phys. B $\mathbf{3 2 4}$ (1989) 581 [INSPIRE].

[31] J. Cardy and P. Calabrese, Unusual corrections to scaling in entanglement entropy, J. Stat. Mech. 04 (2010) P04023 [arXiv: 1002.4353] [INSPIRE].

[32] M.R. Douglas, Conformal field theory techniques in large- $N$ Yang-Mills theory, hep-th/9311130 [INSPIRE].

[33] R. Dijkgraaf, Chiral deformations of conformal field theories, Nucl. Phys. B 493 (1997) 588 [hep-th/9609022] [INSPIRE].

[34] M.R. Gaberdiel, K. Jin and E. Perlmutter, Probing higher spin black holes from CFT, JHEP 10 (2013) 045 [arXiv: 1307.2221] [inSPIRE]. 
[35] S. Datta, J.R. David, M. Ferlaino and S.P. Kumar, Higher spin entanglement entropy from CFT, JHEP 06 (2014) 096 [arXiv: 1402.0007] [INSPIRE].

[36] S. Datta, J.R. David, M. Ferlaino and S.P. Kumar, Universal correction to higher spin entanglement entropy, Phys. Rev. D 90 (2014) 041903 [arXiv:1405.0015] [INSPIRE].

[37] J. Long, Higher spin entanglement entropy, JHEP 12 (2014) 055 [arXiv:1408.1298] [INSPIRE].

[38] S. Datta, J.R. David and S.P. Kumar, Conformal perturbation theory and higher spin entanglement entropy on the torus, JHEP 04 (2015) 041 [arXiv:1412.3946] [INSPIRE].

[39] S. Jackson, L. McGough and H. Verlinde, Conformal bootstrap, universality and gravitational scattering, arXiv:1412.5205 [INSPIRE].

[40] P. Caputa and A. Veliz-Osorio, Entanglement constant for conformal families, Phys. Rev. D 92 (2015) 065010 [arXiv: 1507.00582] [INSPIRE]. 